\title{
CHERN CLASSES OF CONFORMAL BLOCKS
}

\author{
NAJMUDDIN FAKHRUDDIN
}

\begin{abstract}
We derive a formula for the Chern classes of the bundles of conformal blocks on $\overline{\mathrm{M}}_{0, n}$ associated to simple finite dimensional Lie algebras and explore its conseqences in more detail for $\mathfrak{g}=\mathfrak{s l}_{2}$ and for arbitrary $\mathfrak{g}$ and level 1 . We also give a method for computing the first Chern class of such bundles on $\overline{\mathrm{M}}_{g, n}$ for $g>0$.
\end{abstract}

\section{INTRODUCTION}

The mathematical theory of conformal blocks of Tsuchiya-Kanie [21] and Tsuchiya-UenoYamada [22] gives rise to a family of vector bundles, parametrised by a simple Lie algebra $\mathfrak{g}$, a non-negative integer $\ell$ called the level, and an $n$-tuple of dominant weights for $\mathfrak{g}$ of "level $\ell$ ", on $\overline{\mathrm{M}}_{g, n}$, the moduli stack of stable $n$-pointed curves of genus $g$. The ranks of these bundles are given by the celebrated Verlinde formula (see [19] for a survey); the purpose of this article is to investigate the Chern classes of these bundles, with an emphasis on $c_{1}$ or the determinant bundle.

These bundles of conformal blocks have been objects of interest to algebraic geometers ever since it was realised that they can be described in terms of sections of natural line bundles on suitable moduli stacks of parabolic principal bundles on curves. However, our motivation for studying these bundles is the hope that we will get some insight into the geometry of the moduli stacks $\overline{\mathrm{M}}_{g, n}$ themselves, especially when $g=0$. The reason for this is that the bundles of conformal blocks on $\overline{\mathrm{M}}_{0, n}$ are generated by global sections, hence their Chern classes are all nef. In particular, considering the first Chern classes of these bundles we get a large collection of elements in the cone of nef divisors of $\overline{\mathrm{M}}_{0, n}$ and our goal is to describe the classes so obtained.

Our main result, Theorem 3.2, is a formula for the Chern classes of the bundles of conformal blocks on $\overline{\mathrm{M}}_{0, n}$. As a special case of this we obtain explicit divisors representing the first Chern class, Corollary 3.4, as well as a formula for its degree on any F-curve. We refer to $\S 3.2$ for the precise statements of these results which are given in terms of ranks of auxiliary bundles of conformal blocks, which may be computed using the Verlinde formula, and the eigenvalues of the Casimir operator of $\mathfrak{g}$ acting on finite dimensional representations of level $\ell$. The essential ingredients that we use are:

- The KZ connection on the restriction of these bundles to $\mathrm{M}_{0, n}$,

- a formula for the residue of this connection along the boundary divisors and

- a formula of Esnault and Verdier from [4] which allows one to compute the Chern classes of a vector bundle on a smooth projective variety in terms of the residues of a logarithmic connection.

For $\mathfrak{g}=\mathfrak{s l}_{2}$, we show that the non-zero determinants of conformal blocks of level 1 form a basis of $\operatorname{Pic}\left(\overline{\mathrm{M}}_{0, n}\right)_{\mathbb{Q}}$ (Theorem 4.3). For what we call the critical level, we show that the bundles are pulled back from suitable GIT quotients $\left(\mathbb{P}^{1}\right)^{n} / / S L_{2}$ (Theorem 4.5); in general 
they are pulled back from suitable moduli spaces of weighted stable curves constructed by Hassett (Proposition 4.7).

We also specialize our results to the case of an arbitrary simple Lie algebra $\mathfrak{g}$ and $\ell=1$. A particularly interesting case is that of $\mathfrak{s l}_{m}$; the critical level $\mathfrak{s l}_{2}$ determinants reappear here for suitable choices of weights and it seems likely that all level 1 conformal blocks have a GIT interpretation. This has been shown to be true for symmetric weights by N. Giansiracusa [9]. The case of $\mathfrak{s p}_{2 \ell}$ is also noteworthy; the bundles of conformal blocks for these Lie algebras at level 1 turn out to be related to $\mathfrak{s l}_{2}$ conformal blocks at level $\ell$. This gives rise to certain F-nef divisors on $\overline{\mathrm{M}}_{0, n}$ which we do not know are nef.

For $g>0$ one may, in principal, use a method similar to the $g=0$ case for computing the Chern classes in cohomology. However, this is more involved since the bundles of conformal blocks do not have a flat connection - the first Chern class of these bundles restricted to $\overline{\mathrm{M}}_{g, n}$ is in general non-trivial - so we do not work out the details here and restrict ourselves to computing the first Chern class. Basic properties of the moduli stacks and conformal blocks reduce this to the case of $\overline{\mathrm{M}}_{1,1}$ in which case we give an explicit formula in Theorem 6.1. We see from this that for $g>0$ the first Chern class is almost never nef. An exception, perhaps the only one, is the case of $\mathfrak{e}_{8}$ and level 1 in which case the bundle of conformal blocks is the fourth tensor power of the Hodge bundle. Finally, we note that a formula for the Chern classes of the conformal blocks bundles restricted to $\mathrm{M}_{g, n}$ can be obtained from the results of Tsuchimoto [20].

The paper is structured as follows: In $\S 2$ we recall some of the properties of conformal blocks that we shall need, mostly without proof but we give some details in cases where we do not know precise references. In $\S 3$ we derive our main formula for the Chern classes in genus 0 and deduce some corollaries. In $\S 4$ we consider the case of $\mathfrak{s l}_{2}$ and in $\S 5$ that of the general level 1 case in more detail. We then consider the higher genus case in $\S 6$ and conclude the paper in $\S 7$ by discussing some questions.

1.1. Notation. We will work over an arbitrary field $k$ of characteristic zero. $\mathfrak{g}$ will always denote a finite dimensional split simple Lie algebra over $k, \mathfrak{h} \subset \mathfrak{g}$ a Cartan subalgebra, $\Delta \subset \mathfrak{h}^{*}$ the corresponding root system and $\alpha_{1}, \alpha_{2}, \ldots, \alpha_{r}$ a basis of the root system inducing a partition $\Delta=\Delta^{+} \cup \Delta^{-}$. Let $P \subset \mathfrak{h}^{*}$ be the weight lattice and $P_{+} \subset P$ be the set of dominant weights. For $\lambda \in P_{+}$we let $V_{\lambda}$ denote the corresponding irreducible representation of $\mathfrak{g}$. Let $\theta \in \Delta^{+}$be the highest root and let $\mathfrak{s} \cong \mathfrak{s l}_{2}$ be the subalgebra of $\mathfrak{g}$ generated by $H_{\theta}$ and $H_{-\theta}$, where $H_{\alpha}$, for a root $\alpha$, is the corresponding coroot.

We normalize the Killing form $(\mid)$ on $\mathfrak{g}$ so that $(\theta \mid \theta)=2$ and use the same notation for the induced forms on $\mathfrak{h}$ and $\mathfrak{h}^{*}$. We let $\left\{X_{\kappa}\right\}_{\kappa}$ be an orthonormal basis of $\mathfrak{g}$ with respect to this form. Let $h^{\vee}$ be the dual Coxeter number of $\mathfrak{g}$; this is half the scalar by which the normalised Casimir element $\Omega:=\sum_{\kappa} X_{\kappa} \circ X_{\kappa}$ acts on the adjoint representation of $\mathfrak{g}$. For any $\lambda \in P_{+}$, we let $c(\lambda)$ be the scalar by which $\Omega$ acts on $V_{\lambda}$; this is equal to $(\lambda \mid \lambda+2 \rho)$, where $\rho$ is half the sum of the positive roots. For $\ell$ a non-negative integer, let $P_{\ell}=\left\{\lambda \in P_{+} \mid(\lambda \mid \theta) \leq \ell\right\}$. For $\lambda \in P_{+}$, let $\lambda^{*}$ be the highest weight of $\left(V_{\lambda}\right)^{*}$; if $\lambda \in P_{\ell}$ then $\lambda^{*} \in P_{\ell}$ as well.

We shall add $\mathfrak{g}$ as a subscript or superscript to any of the above notation, or notation introduced later, if it is necessary to make $\mathfrak{g}$ explicit.

1.2. Acknowledgements. I thank Patrick Brosnan, Sreedhar Dutta, Norbert Hoffmann, Arvind Nair and Madhav Nori for helpful discussions. Discussions with Valery Alexeev about his work with Swinarksi and also the work of Hassett were very useful and I am grateful to 
him for them. I am particulary indebted to Prakash Belkale for a long correspondence which helped me to learn about conformal blocks and also for his comments on this paper.

A part of this work was done while I was visiting Université Paris-Sud (Orsay) supported by the project "ARCUS". I thank Laurent Clozel for the invitation and Université Paris-Sud for its hospitality.

\section{Conformal BLOCKS}

In this section we recall and reformulate some of the basic definitions and results in the theory of conformal blocks that we shall need later. The original references are [21] and [22] but we shall use [23] and [16] to which we shall refer for most proofs.

2.1. Construction. Let $S$ be a smooth variety over $k$ and $\pi: \mathcal{C} \rightarrow S$ be a proper flat family of curves with only ordinary double point singularities; we do not assume that the fibres of $\pi$ are connected. Let $\bar{p}=\left(p_{1}, \ldots, p_{n}\right)$, with $p_{i}: S \rightarrow \mathcal{C}$ sections of $\pi$ whose images are disjoint and contained in the smooth locus of $\pi$. We also assume that $\mathcal{C} \backslash \cup_{i} p_{i}(S)$ is affine over $S$. Let $\bar{\lambda}=\left(\lambda_{1}, \ldots, \lambda_{n}\right)$ be an $n$-tuple of elements of $P_{\ell}$. To this data is attached a canonically defined locally free sheaf $V_{\mathcal{C}}(\bar{p}, \bar{\lambda})$ on $S$, called the sheaf of covacua [22], [23], [19] or, as we shall call them, conformal blocks [16]. We briefly recall the construction:

Let $\hat{\mathfrak{g}}$ be the affine Lie algebra defined by

$$
\hat{\mathfrak{g}}:=(\mathfrak{g} \otimes k((\xi))) \oplus k \cdot \mathrm{c}
$$

where $\mathrm{c}$ is in the centre of $\hat{\mathfrak{g}}$ and the bracket is defined by

$$
[X \otimes f(\xi), Y \otimes g(\xi)]=[X, Y] \otimes f(\xi) g(\xi)+(X, Y) \operatorname{Res}(g(\xi) d f(\xi)) \cdot c .
$$

Put

$$
\hat{\mathfrak{g}}_{+}=\mathfrak{g} \otimes k[[\xi]] \xi, \quad \hat{\mathfrak{p}}_{+}=\hat{\mathfrak{g}}_{+} \oplus \mathfrak{g} \oplus k \cdot \mathrm{c} .
$$

For $\lambda \in P_{\ell}$, we extend the action of $\mathfrak{g}$ on $V_{\ell}$ to an action of $\hat{\mathfrak{p}}_{+}$by setting

- $\mathrm{c} v=\ell v$ for all $v \in V_{\lambda}$

- $a v=0$ for all $a \in \hat{\mathfrak{g}}_{+}$and $v \in V_{\lambda}$.

Put

$$
\mathcal{M}_{\lambda}:=U(\hat{\mathfrak{g}}) \otimes_{U\left(\hat{\mathfrak{p}}_{+}\right)} V_{\lambda} .
$$

This is a representation of $\hat{\mathfrak{g}}$ and has a unique maximal proper submodule $\mathcal{I}_{\lambda}$. Then

$$
\mathcal{H}_{\lambda}:=\mathcal{M}_{\lambda} / \mathcal{I}_{\lambda}
$$

is an irreducible $\hat{\mathfrak{g}}$ module.

For an integer $n>0$, let

$$
\hat{\mathfrak{g}}_{n}:=\mathfrak{g} \otimes\left(\bigoplus_{i=1}^{n} k\left(\left(\xi_{i}\right)\right)\right) \oplus k \cdot c .
$$

This has a natural Lie algebra structure making it into a quotient of $(\hat{\mathfrak{g}})^{n}$.

For $\bar{\lambda} \in P_{\ell}^{n}$, let

$$
\mathcal{H}_{\bar{\lambda}}:=\bigotimes_{i=1}^{n} \mathcal{H}_{\lambda_{i}}
$$

This is an irreducible representation of $\hat{\mathfrak{g}}_{n}$; the natural action of $(\hat{\mathfrak{g}})^{n}$ factors through $\hat{\mathfrak{g}}_{n}$. 
We first assume that $S$ is affine, so $S=\operatorname{Spec}(A)$ for some $k$-algebra $A$, and we also assume that there are given isomorphisms $\eta_{i}: \widehat{\mathcal{O}}_{\mathcal{C}, p_{i}(S)} \rightarrow A[[\xi]]$ for each $i$. Let $B=\Gamma\left(\mathcal{C}-\cup_{i=1}^{n} p_{i}(S)\right)$. For each $i$, using the isomorphism $\eta_{i}$, we get a map $B \rightarrow A\left(\left(\xi_{i}\right)\right)$ which induces injections

$$
\mathfrak{g} \otimes_{k} B \rightarrow \mathfrak{g} \otimes_{k}\left(\bigoplus_{i=1}^{n} k\left(\left(\xi_{i}\right)\right)\right) \otimes_{k} A \rightarrow \hat{\mathfrak{g}}_{n} \otimes_{k} A
$$

The fact that the sum of the residues of a 1-form on a smooth projective curve is zero implies that this injection makes $\mathfrak{g} \otimes_{k} B$ into a sub Lie algebra of $\hat{\mathfrak{g}}_{n} \otimes_{k} A$.

By linearity $\mathcal{H}_{\bar{\lambda}} \otimes_{k} A$ is a representation of $\hat{\mathfrak{g}}_{n} \otimes_{k} A$ and the sheaf of conformal blocks $V_{\mathcal{C}}(\bar{p}, \bar{\lambda})$ is defined to be the quasi-coherent sheafon $S$, which is in fact locally free of finite rank, corresponding to the $A$-module

$$
\mathcal{H}_{\bar{\lambda}} \otimes_{k} A /\left(\mathfrak{g} \otimes_{k} B\right) \cdot\left(\mathcal{H}_{\bar{\lambda}} \otimes_{k} A\right) .
$$

One may give an intrinsic description of this module without using the isomorphisms $\eta_{i}$; we only use this implicitly so we refer the reader to [16] for the details. Since such isomorphisms always exist Zariski locally on any $S$, we may sheafify the above construction to define a locally free sheaf $V_{\mathcal{C}}(\bar{p}, \bar{\lambda})$ for general $S$.

Note that if $\mathcal{C}=\mathcal{C}^{\prime} \cup \mathcal{C}^{\prime \prime}$ is a disjoint union of two families of semi-stable curves over $S$ then we have $V_{\mathcal{C}}(\bar{p}, \bar{\lambda}) \cong V_{\mathcal{C}^{\prime}}\left(\bar{p}^{\prime}, \bar{\lambda}^{\prime}\right) \otimes V_{\mathcal{C}^{\prime \prime}}\left(\bar{p}^{\prime \prime}, \bar{\lambda}^{\prime \prime}\right)$, where $\bar{p}^{\prime}$ denotes the tuple of sections lying in $\mathcal{C}^{\prime}, \bar{\lambda}^{\prime}$ denotes the corresponding tuple of weights and similarly for $\bar{p}^{\prime \prime}$ and $\bar{\lambda}^{\prime \prime}$.

\section{sec:basic 2.2. Basic properties.}

Keeping the previous notation, let $\bar{q}=\left(q_{1}, \ldots, q_{m}\right)$ with the $q_{j}$ also sections of $\pi$ with images which are disjoint, disjoint from all the $p_{i}(S)$ and also contained in the smooth locus of $\pi$. Let $\overline{p q}=\left(p_{1}, \ldots, p_{n}, q_{1}, \ldots, q_{m}\right), \overline{\lambda 0_{m}}=\left(\lambda_{1}, \ldots, \lambda_{n}, 0, \ldots, 0\right)$, where we have $m$ zeros. We then have the following [19, 2.3.2], [16, Proposition 3.4]:

prop:propProposition 2.1 (Propagation of vacua). There is a natural isomorphism $V_{\mathcal{C}}(\bar{p}, \bar{\lambda}) \stackrel{\sim}{\longrightarrow}$ $V_{\mathcal{C}}\left(\overline{p q}, \overline{\lambda 0_{m}}\right)$. Moreover, these isomorphisms are compatible as $m$ varies.

We now drop the condition that $\mathcal{C} \backslash \cup_{i} p_{i}(S)$ is affine over $S$. For $S^{\prime} \rightarrow S$ any morphism, we let $\mathcal{C}^{\prime}=\mathcal{C} \times{ }_{S} S^{\prime}, \pi^{\prime}: \mathcal{C}^{\prime} \rightarrow S^{\prime}$ the induced morphism and $p_{i}^{\prime}$ the induced sections. We may find an etale cover $S^{\prime} \rightarrow S$ and $m$ sections $\overline{q^{\prime}}=\left(q_{1}^{\prime}, \ldots, q_{m}^{\prime}\right)$ of $\pi^{\prime}$ as above so that $\mathcal{C}^{\prime} \backslash\left(\cup_{i} p_{i}^{\prime}\left(S^{\prime}\right) \cup_{j} q_{j}^{\prime}\left(S^{\prime}\right)\right)$ is affine. Therefore, $V_{\mathcal{C}^{\prime}}\left(\overline{p^{\prime} q^{\prime}}, \overline{\lambda 0_{m}}\right)$ is defined and is a locally free sheaf on $S^{\prime}$. By Proposition 2.1 we get natural descent data for this sheaf with respect to the morphism $S^{\prime} \rightarrow S$ and we define $V_{\mathcal{C}}(\bar{p}, \bar{\lambda})$ to be the descent of this sheaf to $S$.

To see that this is independent of the choice of the etale cover $S^{\prime} \rightarrow S$ and sections $q_{j}^{\prime}$ we use the same proposition. Note that it may happen that if we use two sets of auxiliary sections then all the sections in the union may not be pairwise disjoint. However, since the sections are disjoint or equal at all generic points of the base $S$, we get get canonical isomorphisms over a dense open set of $S$. Using further auxiliary sections disjoint from all the original ones, etale locally around each point of $S$, one sees that these isomorphisms extend over all of $S$.

Since the sheaves of conformal blocks are compatible with base change, the above discussion shows that for any $n$-tuple $\bar{\lambda} \in P_{\ell}{ }^{n}$ one has a well defined sheaf of conformal blocks on $\overline{\mathrm{M}}_{g, n}$, the moduli stack of stable $n$-pointed curves of genus $g$. 
Let $\bar{p}, \bar{\lambda}$ and $\bar{q}$ be as above with $m=2$. Let $\pi_{\mathcal{D}}: \mathcal{D} \rightarrow S$ be the family of curves obtained by gluing $\mathcal{C}$ along the sections $q_{1}$ and $q_{2}$. The sections $p_{i}$ induce sections of $\pi_{\mathcal{D}}: \mathcal{D} \rightarrow S$ which we also denote by $p_{i}$. For $\mu \in P_{\ell}$, let $\overline{\lambda \mu}=\left(\lambda_{1}, \ldots, \lambda_{n}, \mu, \mu^{*}\right)$. We then have [16, Proposition 4.1], [19, 2.4.2]

Proposition 2.2 (Factorisation formula). There are natural isomorphisms

$$
V_{\mathcal{D}}(\bar{p}, \bar{\lambda}) \stackrel{\sim}{\longrightarrow} \bigoplus_{\mu \in P_{\ell}} V_{\mathcal{C}}(\overline{p q}, \overline{\lambda \mu})
$$

Let $\pi: \mathcal{C} \rightarrow S$ be a family of semi-stable curves and $\bar{p}=\left(p_{1}, \ldots, p_{n+1}\right)$ an $n+1$-tuple of sections making $(\mathcal{C}, \bar{p})$ into a family of stable $n+1$-pointed curves for some $n \geq 0$. Let $\bar{\lambda}=\left(\lambda_{1}, \ldots, \lambda_{n}, 0\right) \in P_{\lambda}{ }^{n+1}$. If we let $\bar{p}^{\prime}=\left(p_{1}, \ldots, p_{n}\right)$, then the family of $n$ pointed curves $\left(\mathcal{C}, \bar{p}^{\prime}\right)$ may no longer be stable. We have a stablisation morphism $\sigma: \mathcal{C} \rightarrow \mathcal{C}^{\prime}$ over $S$ which contracts certain smooth rational curves in each fibre of $\pi$ so that $\left(\mathcal{C}^{\prime}, \bar{p}^{\prime}\right)$ is a stable family of $n$-pointed curves. Set $\bar{\lambda}^{\prime}=\left(\lambda_{1}, \ldots, \lambda_{n}\right)$.

Lemma 2.3. With notation as above, there is a natural isomorphism of sheaves

$$
V_{\mathcal{C}^{\prime}}\left(\bar{p}^{\prime}, \bar{\lambda}^{\prime}\right) \stackrel{\sim}{\longrightarrow} V_{\mathcal{C}}(\bar{p}, \bar{\lambda}) \text {. }
$$

Proof. The stabilisation morphism $\sigma$ induces a morphism $\mathcal{C} \backslash \cup_{i=1}^{n+1} p_{i}(S) \rightarrow \mathcal{C}^{\prime} \backslash \cup_{i=1}^{n} p_{i}(S)$ which gives rise to the map of sheaves. Since the bundles of conformal blocks are locally free, we may check that this map is an isomorphism fibrewise.

There are two types of rational curve components that may get contracted by the stabilisation morphism. One type is a smooth rational curve with two marked points, one of them being the $n+1$ 'st and intersecting the union of the other components in a single point. When this is contracted the point of intersection becomes the other marked point. The other type is a smooth rational curve meeting the union of the other components in two points and containing the $n+1$ 'st marked point, this being the unique marked point on it.

In both these cases, a direct application of the factorisation formula shows that the map is indeed an isomorphism.

As a consequence of the above we see the the bundles of conformal blocks are compatible with the natural morphisms among the moduli stacks of stable curves. More precisely, we have

Proposition 2.4. For $\bar{\lambda} \in P_{\ell}^{n}$, let $\mathbb{V}_{g, n, \bar{\lambda}}$ denote the sheaf of conformal blocks associated to $\bar{\lambda}$ on $\overline{\mathrm{M}}_{g, n}$.

(1) Let $f_{i}: \overline{\mathrm{M}}_{g, n} \rightarrow \overline{\mathrm{M}}_{g, n-1}$ be the morphism given by forgetting the $i$ 'th marked point. If $\lambda_{i}=0$ for some $i$, then $\mathbb{V}_{g, n, \bar{\lambda}} \cong f_{i}^{*}\left(\mathbb{V}_{g, n, \bar{\lambda}^{\prime}}\right)$, where $\bar{\lambda}^{\prime}$ is obtained from $\bar{\lambda}$ by deleting $\lambda_{i}$

(2) Let $\gamma: \overline{\mathrm{M}}_{g_{1}, n_{1}+1} \times \overline{\mathrm{M}}_{g_{2}, n_{2}+1} \rightarrow \overline{\mathrm{M}}_{g_{1}+g_{2}, n_{1}+n_{2}}$ be the gluing morphism, where we glue along the last marked point for each factor. Then for $\bar{\lambda} \in P_{\ell}^{n_{1}+n_{2}}$ we have a natural isomorphism

$$
\gamma^{*}\left(\mathbb{V}_{g_{1}+g_{2}, n_{1}+n_{2}, \bar{\lambda}}\right) \stackrel{\sim}{\rightarrow} \bigoplus_{\mu \in P_{\ell}} \mathbb{V}_{g_{1}, n_{1}+1, \bar{\lambda}^{1} \mu} \otimes \mathbb{V}_{g_{2}, n_{2}+1, \bar{\lambda}^{2} \mu^{*}}
$$

where $\bar{\lambda}^{1} \mu:=\left(\lambda_{1}, \ldots, \lambda_{n_{1}}, \mu\right)$ and $\bar{\lambda}^{2} \mu^{*}:=\left(\lambda_{n_{1}+1}, \ldots, \lambda_{n_{1}+n_{2}}, \mu^{*}\right)$. 
(3) Let $\gamma: \overline{\mathrm{M}}_{g-1, n+2} \rightarrow \overline{\mathrm{M}}_{g, n}$ be the gluing morphism where we glue the last two marked points to each other. Then for $\bar{\lambda} \in P_{\ell}^{n}$ we have an isomorphism

$$
\gamma^{*}\left(\mathbb{V}_{g, n, \bar{\lambda}}\right) \stackrel{\sim}{\rightarrow} \bigoplus_{\mu \in P_{\ell}} \mathbb{V}_{g-1, n+2, \overline{\lambda \mu}}
$$

where $\overline{\lambda \mu}:=\left(\lambda_{1}, \ldots, \lambda_{n}, \mu, \mu^{*}\right)$.

Proof. (1) follows from Lemma 2.3 and (2), (3) follow from Proposition 2.2

In this paper we shall mostly be concerned with the case $g=0$. In this case, $\overline{\mathrm{M}}_{0, n}$ is a smooth projective variety and we shall from now on denote the locally free sheaf $\mathbb{V}_{0, n, \bar{\lambda}}$ on $\overline{\mathrm{M}}_{0, n}$ simply by $\mathbb{V}_{\bar{\lambda}}$, its determinant line bundle by $\mathbb{D}_{\bar{\lambda}}$ and its rank by $r_{\bar{\lambda}}$.

Lemma 2.5. All the $\mathbb{V}_{\bar{\lambda}}$ are generated by their global sections, therefore so are all the $\mathbb{D}_{\bar{\lambda}}$. In particular, the $\mathbb{D}_{\bar{\lambda}}$ are nef line bundles.

Proof. With notation as in the beginning of this section, by the construction of the sheaves of conformal blocks there is always a natural map from the constant vector bundle on $S$ with fibre $\left(\otimes_{i} V_{\lambda_{i}}\right)_{\mathfrak{g}}$ to $V_{\mathcal{C}}(\bar{p}, \bar{\lambda})$ which is induced by the inclusion of $V_{\lambda}$ in $\mathcal{H}_{\lambda}$. It follows from [23, Proposition 3.5.1] that this map is surjective if the fibres of the family are smooth curves of genus 0 . The proof only uses the fact that given any point $x \in \mathbb{P}^{1}(k)$ there exists $f \in \Gamma\left(\mathbb{P}^{1}-\{x\}, \mathcal{O}_{\mathbb{P}^{1}-\{x\}}\right)$ which has a simple pole at $x$. If $C$ is a semi-stable curve of genus 0 and $x \in C(k)$ is a smooth point, then there exists $f \in \Gamma\left(C-\{x\}, \mathcal{O}_{C-\{x\}}\right)$ having a simple pole at $x$, so the proof of [23, Proposition 3.5.1] extends to the case where the fibres are semi-stable curves of genus 0 .

Remark 2.6. The map from $\left(\otimes_{i} V_{\lambda_{i}}\right)_{\mathfrak{g}}$ to $V_{\mathcal{C}}(\bar{p}, \bar{\lambda})$ is in general not surjective if the fibres have genus $g>0$. In fact, in this case it follows from Theorem 6.1 that the determinants of conformal blocks on $\overline{\mathrm{M}}_{0, n}$ are often not even nef.

Recall that $\overline{\mathrm{M}}_{0, n} \backslash \mathrm{M}_{0, n}$ is a divisor with simple normal crossings, so $\overline{\mathrm{M}}_{0, n}$ has a natural stratification by smooth strata. Keel [13] has shown that the Chow groups of $\overline{\mathrm{M}}_{0, n}$ are generated by the classes of the closures of the (irreducible componenents) of the strata and the Chow groups are equal to the Chow groups modulo numerical equivalence. Thus, one way of computing the class of $\mathbb{D}_{\bar{\lambda}}$ in $\operatorname{Pic}\left(\overline{\mathrm{M}}_{0, n}\right)$ is by computing the degree of $\mathbb{V}_{\bar{\lambda}}$ restricted to the one dimensional strata, called vital curves in [14].

Classes of vital curves modulo numerical equivalence correspond to partitions $\{1, \ldots, n\}=$ $\sqcup_{k=1}^{4} N_{k}$, with $\left|N_{k}\right|=n_{k}>0$. Given such a partition, let $F$ be the family of $n$-pointed genus 0 curves given by gluing, for each $k=1,2,3,4$, a fixed $n_{k}+1$-pointed curve $C_{k}$ of genus 0 at the last marked point along the $k$-th section of the universal family over $\overline{\mathrm{M}}_{0,4}$. If $n_{k}=1$ for some $k$, we do not glue any curve at the $k$ 'th section. This gives rise to a family of stable $n$-pointed curves of genus 0 such that the class in the Chow group of the image of $\overline{\mathrm{M}}_{0,4}$ in $\overline{\mathrm{M}}_{0, n}$ by the classifying map for this family is independent of the choice of the glued curves.

Given a partition as above and $\bar{\mu}=\left(\mu_{1}, \mu_{2}, \mu_{3}, \mu_{4}\right) \in P_{\ell}^{4}$, let $\bar{\lambda} \mu_{k}^{*}$ be the $n_{k}+1$-tuple $\left(\lambda_{i_{1}}, \ldots, \lambda_{i_{n_{k}}}, \mu_{k}^{*}\right)$ where $N_{k}=\left\{i_{1}, i_{2}, \ldots, i_{n_{k}}\right\}$. Since in the construction of $F$ the attached curves do not vary in moduli, it follows from the factorisation formula applied four times that we have 
Proposition 2.7.

$$
\operatorname{deg}\left(\left.\mathbb{V}_{\bar{\lambda}}\right|_{F}\right)=\sum_{\bar{\mu} \in P_{\ell}{ }^{4}} \operatorname{deg}\left(\mathbb{V}_{\bar{\mu}}\right) \prod_{k=1}^{4} r_{\bar{\lambda} \mu_{k}^{*}}
$$

The ranks of the bundles of conformal blocks can be computed from the Verlinde formula or inductively from the case $n=3$, so it follows that to compute the determinant of $\mathbb{V}_{\bar{\lambda}}$, equivalently its degree on any vital curve, it suffices to consider the $n=4$ case.

Remark 2.8. For arbitrary $g$, the closures of the 1-dimensional strata of $\overline{\mathrm{M}}_{g, n}$ are called F-curves and there is a simple way of enumerating all these [10, Theorem 2.2]. It is known that their classes generate $N_{1}\left(\overline{\mathrm{M}}_{g, n}\right)_{\mathbb{Q}}$, the space of 1-cycles modulo numerical equivalence, so it follows from the description of F-curves and the factorisation formula that to compute $c_{1}\left(\mathbb{V}_{g, n, \bar{\lambda}}\right)$ for all $g, n$ it suffices to be able to compute it in the cases $(g, n)=(0,4)$ or $(1,1)$.

2.3. The KZ/Hitchin/WZW connection. The sheaves of conformal blocks associated to a smooth family of curves have a natural flat projective connection called the WZW or Hitchin connection; this is one of the main ingredients in our computation of the Chern classes. However, for that purpose it is important to lift this to a flat connection. For arbitrary $g$ this is not always possible globally but it is for $g=0$ or 1 . The lift is not canonical and depends on some auxiliary choices and our first goal in this section is to understand this dependence explicitly.

For the sake of simplicity, we assume that $S$ is affine and $A=\Gamma\left(S, \mathcal{O}_{S}\right)$ as before. Then the first choice is that of formal parameters, i.e., the isomorphisms $\eta_{i}$, along each section of the family of curves $\pi: \mathcal{C} \rightarrow S$ with an $n$-tuple of sections $\bar{p}$. The second is that of a suitable symmetric bidifferential $\omega$ on the family, see [23, p. 16]. Given this data, the action of a vector field $D$ on $S$ on is defined as follows [23, Section 5.1]: We first lift $D$ to a vector field, also denoted by $D$, on $\mathcal{C} \backslash \cup_{i} p_{i}(S)$. For each $i$, we write $\left(\eta_{i}^{-1}\right)^{*}(D)=D_{i, h o r}+D_{i, v e r t}$ where $D_{i, h o r}(\xi)=0$ and $D_{i, v e r t}$ kills $A$. Then we have the Sugawara operators $T\left(D_{i, v e r t}\right)$ which act on $\mathcal{H}_{\lambda_{i}}$ and hence on $\mathcal{H}_{\bar{\lambda}}$ by acting on the $i$ 'th factor. Morevoer, the bidifferential $\omega$ gives elements $a_{\omega, i}\left(D_{i, v e r t}\right) \in A[23,(5.4)]$ whose sum over all $i$ we write simply as $a_{\omega}\left(D_{\text {vert }}\right)$; we do not recall the full definition here but note that it is of the form $\frac{\ell \operatorname{dimg}}{\ell+h^{\vee}} f$, with $f \in A$ not depending on any data associated to $\mathfrak{g}$.

The action of $D$ on $V_{\mathcal{C}}(\bar{p}, \bar{\lambda})$, which we denote by $\nabla_{D}$, is induced by the action on $\mathcal{H}_{\bar{\lambda}} \otimes_{k} A$ given by

$$
\nabla_{D}(v \otimes f)=D_{h o r}(v \otimes f)+\left(\sum_{i=1}^{n} T\left(D_{i, v e r t}\right)(v)\right) \otimes f-v \otimes a_{\omega}\left(D_{v e r t}\right) \cdot f,
$$

where $v \in \mathcal{H}_{\bar{\lambda}}$ and $f \in A$ and the action of $D_{\text {hor }}$ is given by coordinatewise differentiation, i.e., it acts on the $A$ component of $\mathcal{H}_{\bar{\lambda}} \otimes_{k} A$. We note that the energy-momentum tensor $T$, whose definition we recall below, as well as the operator $a_{\omega}$ depend on the choice of parameters. Moreover, the first term also depends on this choice since this is implicit in the tensor product decomposition.

We now choose some different isomorphisms $\eta_{i}^{\prime}$ and compute how the connection changes; in the following we denote all terms defined using these new isomorphisms with a '. By definition, the difference of the two actions $\nabla_{D}-\nabla_{D}^{\prime}$ is given by the operator

$$
D_{h o r}-D_{h o r}^{\prime}+T\left(D_{v e r t}\right)-T^{\prime}\left(D_{v e r t}^{\prime}\right)-\left(a_{\omega}\left(D_{v e r t}\right)-a_{\omega}^{\prime}\left(D_{v e r t}^{\prime}\right)\right)
$$


where to simplify the notation we have suppressed the sum over $i$. Adding and subtracting suitable terms, this is equal to

$D_{\text {hor }}-D_{\text {hor }}^{\prime}+T\left(D_{\text {vert }}-D_{\text {vert }}^{\prime}\right)+T\left(D_{\text {vert }}^{\prime}\right)-T^{\prime}\left(D_{\text {vert }}^{\prime}\right)-\left(a_{\omega}\left(D_{\text {vert }}-D_{\text {vert }}^{\prime}\right)+a_{\omega}\left(D_{\text {vert }}^{\prime}\right)-a_{\omega}^{\prime}\left(D_{\text {vert }}^{\prime}\right)\right)$.

It follows from the discussion before Lemma 2.13 of [16], more precisely by using the version for integrable representations [23, Lemma 2.13] rather than the Fock representation, that

$$
T\left(D_{\text {vert }}-D_{\text {vert }}^{\prime}\right)=-\left(D_{\text {hor }}-D_{\text {hor }}^{\prime}\right)+U
$$

where $U$ is the operator acting on $\mathcal{H}_{\bar{\lambda}} \otimes_{k} A$ by the scalar (see below), giving the action of $T\left(D_{\text {vert }}-D_{\text {vert }}^{\prime}\right)$ on $\otimes_{i} V_{\lambda_{i}} \subset \mathcal{H}_{\bar{\lambda}}$.

Using the definition in $[23,(5.4)]$ one sees that $a_{\omega}\left(D_{\text {vert }}-D_{\text {vert }}^{\prime}\right)=0$. The change of variables formula for the energy-momentum tensor $T([6,8.2 .2]$ or Theorem 3.4.3 (2) of [23]) and for the projective connection associated to the bidifferential $\omega$ ([23, Theorem 1.115]) implies that both these terms change by the Schwarzian derivative when they are applied to the same vector field but different coordinates are used. Thus the changes in these terms cancel out.

It follows that the difference in the connections is given simply by the operator $U$. As a function of $\bar{\lambda}$ it depends only on the $c\left(\lambda_{i}\right)$. To see this, we first recall the definition of the energy-momentum tensor $T$.

We use the notation

$$
\begin{aligned}
& X(n)=X \otimes \xi^{n}, X \in \mathfrak{g} \\
& X(z)=\sum_{n \in \mathbb{Z}} X(n) z^{-n-1}
\end{aligned}
$$

The normal ordering :: is defined by

$$
: X(n) Y(m):= \begin{cases}X(n) Y(n) & n<m, \\ \frac{1}{2}(X(n) Y(m)+Y(m) X(n)) & n=m, \\ Y(m) X(n) & n>m .\end{cases}
$$

Put

$$
L_{n}=\frac{1}{2\left(\ell+h^{\vee}\right)} \sum_{m \in \mathbb{Z}} \sum_{\kappa}: X_{\kappa}(m) X_{\kappa}(n-m):
$$

and

$$
T(z)=\sum_{n \in \mathbb{Z}} L_{n} z^{-n-2}
$$

The $L_{n}$ 's are called Virasoro operators and act on $\mathcal{H}_{\lambda}$.

For $D=f(z) \frac{d}{d z}$ with $f \in k((z))$, put

$$
T(D)=\operatorname{Res}_{z=0}(T(z) f(z) d z) .
$$

Then $T(D)$ acts on $\mathcal{H}_{\lambda}$.

If $f=\sum_{j=1}^{\infty} a_{j} z^{j}$ then $T(D)=\sum_{j=1}^{\infty} a_{j} L_{j-1}$. The formula for the $L_{n}$ shows that this preserves $V_{\lambda} \subset \mathcal{H}_{\lambda}$ and acts on it as the operator $\frac{a_{1} \Omega}{2\left(\ell+h^{\vee}\right)}$, hence by multiplication by $\frac{a_{1} c(\lambda)}{2\left(\ell+h^{\vee}\right)}$.

We summarize the above discussion in

Lemma 2.9. (1) For each $i, D_{i, v e r t}-D_{i, v e r t}^{\prime}$ is of the form $f_{i}(\xi) \frac{d}{d \xi}$ with $f(\xi)=\sum_{j=1}^{\infty} a_{j}^{i} \xi^{j}$ and $a_{j}^{i} \in A$. 
(2) $\nabla_{D}-\nabla_{D}^{\prime}$ is given by multiplication by

$$
\left(2\left(\ell+h^{\vee}\right)\right)^{-1} \sum_{i} a_{1}^{i} c_{1}\left(\lambda_{i}\right)
$$

Proof. The first part follows easily from the definitions and the second from the discussion preceding the statement of the lemma.

The connection on the sheaf of conformal blocks has logarithmic singularities along the boundary divisors for a degenerating family of smooth curves. Our formula for the Chern classes will be obtained from considerations of the residues of the connection along the boundary. These are known to have a simple description which we now recall:

Let $C^{\prime}$ be a smooth projective curve with distinct points $q_{1}, q_{2}$ in $C^{\prime}(k)$ and let $C$ be the nodal curve obtained by gluing these points together. We assume that $C$ is connected. Given local parameters $\xi_{q_{1}}$ and $\xi_{q_{2}}$ at $q_{1}$ and $q_{2}$ respectively, there exists a natural smoothening $\pi$ : $\mathcal{C} \rightarrow S$ of $C$. Here $S=\operatorname{Spec}(k[[t]]), \pi$ is proper and flat, the special fibre is $C$ and the generic fibre is smooth. Moreover, if $q$ denotes the node on $C$, the formal completion of $C-\{q\}$ in $\mathcal{C}$ is naturally isomorphic to the formal completion of $C-\{q\}$ in $C-\{q\} \times{ }_{k} \operatorname{Spec}(k[[t]])$. In particular, any smooth point of $C(k)$ extends naturally to a section of $\pi$ and a local parameter for such a point extends to a local equation for the corresponding section. This smoothening can be constructed easily using deformation theory and the Grothendieck existence theorem; we shall give a more explicit description in the cases we actually use.

Now suppose $p_{1}, p_{2}, \ldots, p_{n}$ are smooth rational points on the special fibre $C$ which we extend to sections as described above and let $\bar{\lambda} \in P_{\ell}^{n}$. Let $D$ denote the vector field $t \frac{d}{d t}$ on $S$. We lift $D$ to a rational vector field on $\mathcal{C}$ which is regular outside the sections. It follows from the calculations in [16, Section 4] that the Sugawara action of $D$ induces an operator on $V_{\mathcal{C}}(\bar{p}, \bar{\lambda})$ which restricts to the operator on $V_{C}(\bar{p}, \bar{\lambda})$ given as follows:

Proposition 2.10. Under the natural identification of $V_{C}(\bar{p}, \bar{\lambda})$ with $\oplus_{\mu \in P_{\ell}} V_{C^{\prime}}(\overline{p q}, \overline{\lambda \mu})$ given by the factorisation formula, the operator acts on each summand $V_{C^{\prime}}(\overline{p q}, \overline{\lambda \mu})$ by multiplication by $\frac{c(\mu)}{2\left(\ell+h^{\vee}\right)}+b$ with $b$ being given by the Sugawara action of $D_{0}$, the restriction of $D$ to $C^{\prime}$.

Note that since $D_{0}$ is a vertical vector field, $b$ does not depend on $\mu$ and can be computed using a bidifferential, see [23, Lemma 5.1]. In [16, Theorem 4.5] it appears to be claimed that $b$ is always zero but we do not see why. However, we will see that it is zero in the cases we consider.

Note that in the above we have not included the $a_{\omega}$ term in the action. This will vanish in the case $g=0$ that we consider but not for $g>0$.

\section{THE CASE $g=0$}

In this section we derive our main formulae for the Chern classes in genus 0 by explicitly computing the residues of the $\mathrm{KZ}$ connection on $\mathbb{V}_{\bar{\lambda}}$ along all the boundary divisors in $\overline{\mathrm{M}}_{0, n}$.

3.1. In order to carry out our computations we shall need an explicit description of $\mathrm{M}_{0, n}$ and explicit equations for the $n$ sections. Therefore, we identify $\mathrm{M}_{0, n}$ with the open subset of $\mathbb{A}^{n-3}$ given by

$$
\left\{\left(z_{1}, z_{2}, \ldots, z_{n-3}\right) \in \mathbb{A}^{n-3} \mid z_{i} \neq 0,1 \text { for all } i \text { and } z_{i} \neq z_{j} \text { for } i \neq j\right\} .
$$

The universal family of marked curves is given by $\mathrm{M}_{0, n} \times \mathbb{P}^{1}$ with the $n$ ordered sections given by the $n$ morphisms $\mathrm{M}_{0, n} \rightarrow \mathbb{P}^{1},\left(z_{1}, z_{2}, \ldots, z_{n-3}\right) \mapsto z_{1}, z_{2}, \ldots, z_{n-3}, 0,1, \infty$. Letting $x$ 
be the coordinate on $\mathbb{P}^{1}$, the sections are given by the equations $x=z_{1}, z_{2}, \ldots, z_{n-3}, 0,1$ and $1 / x=0$.

For the rest of this section, we fix a simple Lie algebra $\mathfrak{g}$, a level $\ell$ and $\bar{\lambda}=\left(\lambda_{1}, \lambda_{2}, \ldots, \lambda_{n}\right) \in$ $P_{\ell}^{n}$. If we use the bidifferential $\omega(x, y)=\frac{d x d y}{(x-y)^{2}}$ on $\mathbb{P}^{1} \times \mathbb{P}^{1}$, by [23, Section 5.1] we get a well defined flat connection on $\mathbb{V}_{\bar{\lambda}}$ restricted to $\mathrm{M}_{0, n}$ with regular singularities which we call the KZ connection. In fact, since all equations for the sections are fractional linear and the Schwarzian derivative of such a function is 0 , it follows from the definition $[23,(5.40)]$ that $a_{\omega, i}\left(D_{i, v e r t}\right)=0$ for all $i$ for any vector field $D$ on $\mathrm{M}_{0, n}$. To make things precise, given a vector field $D$ on $\mathrm{M}_{0, n}$ we will always lift it to a vector field on $\mathrm{M}_{0, n} \times \mathbb{P}^{1}$ so that it is constant on the fibres.

We now compute the residues of the $\mathrm{KZ}$ connection along the boundary divisors in $\overline{\mathrm{M}}_{0, n}$. We will first choose good local coordinates in order to be able to apply Proposition 2.10 and then compute the change in the connection, hence the change in the residue, by applying Lemma 2.9. All the local coordinates that we use will be fractional linear so it follows from [23, Lemma 5.1] that the constant $b$ occurring in Proposition 2.10 is always zero. What we need then is to compute the functions $a_{1}^{i}$ occurring in Lemma 2.9.

Recall that the boundary divisors are parametrised by partitions $\{1,2, \ldots, n\}=A \sqcup B$ with $|A|,|B| \geq 2$. In the coordinates above, they correspond to the exceptional divisors in the blowup of the following loci:

(1) For $\emptyset \neq S \subset\{1,2, \ldots, n-3\}$, the locus in $\mathbb{A}^{n-3} \supset \mathrm{M}_{0, n}$ given by the equations $\left\{z_{i}=0\right\}_{i \in S}$.

(2) For $\emptyset \neq S \subset\{1,2, \ldots, n-3\}$, the locus in $\mathbb{A}^{n-3} \supset \mathrm{M}_{0, n}$ given by the equations $\left\{z_{i}=1\right\}_{i \in S}$.

(3) For $\emptyset \neq S \subset\{1,2, \ldots, n-3\}$, the locus in $\left(\mathbb{P}^{1}\right)^{n-3} \supset \mathbb{A}^{n-3} \supset \mathrm{M}_{0, n}$ given by the equations $\left\{1 / z_{i}=0\right\}_{i \in S}$.

(4) For $S \subset\{1,2, \ldots, n-3\}$, with $|S| \geq 2$, the locus in $\mathbb{A}^{n-3} \supset \mathrm{M}_{0, n}$ given by the equations $\left\{z_{i}=z_{j}\right\}_{i, j \in S}$.

Globally, each of these divisors is the image of the embedding of $\overline{\mathrm{M}}_{0, r+2} \times \overline{\mathrm{M}}_{0, n-r}$ into $\overline{\mathrm{M}}_{0, n}$ by a suitable gluing map, where $r=|S|$ in the first three cases and $r=|S|-1$ for the last case. By Proposition 2.2, the restriction of $\mathbb{V}_{\bar{\lambda}}$ to each of these divisors is a sum $\oplus_{\mu \in P_{\ell}} \mathbb{V}_{\bar{\lambda}^{\prime} \mu} \otimes \mathbb{V}_{\bar{\lambda}^{\prime \prime} \mu^{*}}$, where $\bar{\lambda}^{\prime} \mu$ (resp. $\left.\bar{\lambda}^{\prime \prime} \mu^{*}\right)$ is obtained by restricting $\bar{\lambda}$ and attaching $\mu$ (resp. $\mu^{*}$ ) at the glued point of the first (resp. second) component. The residue of the KZ connection along these divisors - this is an endomorphism of the restricted bundle-preserves this direct sum decomposition and moreover acts on each summand by a scalar which we shall now determine.

In what follows, we shall denote $\partial / \partial z_{i}$ by $\partial_{i}$ and $\partial / \partial x$ by $\partial_{x}$.

3.1.1. Boundary divisors of type (1): For ease of notation, we shall assume $S=\{1,2 \ldots, r\}$ since the general case follows from this by permuting coordinates. An open set in the blowup is given by $U \cong \mathbb{A}^{n-3}$ with coordinates $t, w_{2}, \ldots, w_{r}, z_{r+1}, \ldots, z_{n-3}$ with the map to $\mathrm{M}_{0, n}$ given by $\left(t, w_{2}, \ldots, w_{r}, z_{r+1}, \ldots, z_{n-3}\right) \mapsto\left(t, t w_{2}, \ldots, t w_{r}, z_{r+1}, \ldots, z_{n-3}\right)$ and the exceptional divisor $B$ is given by $t=0$. The universal family in a neighbourhood of the generic point of the exceptional divisor is given by blowing up the locus given by $t=x=0$ in $U \times \mathbb{P}^{1}$, so over the generic point there are two components isomorphic to $\mathbb{P}^{1}$ meeting transversally in a single point. 
Let $y:=t / x$. The $n$ sections defined over $\mathrm{M}_{0, n}$ extend to sections of this family as follows, where $w_{1}:=1$ :

- The sections given by $x=z_{r+1} \ldots, z_{n}, x=1$ and $1 / x=0$ are given by the same equations.

- The section given by $x=0$ is given by $1 / y=0$.

- The sections given by $x=z_{i}, 1 \leq i \leq r$, are given by $y=w_{i}^{-1}$.

Replacing $k$ by $k\left(w_{2}, \ldots, w_{r}, z_{r+1}, \ldots, z_{n-1}\right)$, it follows from Proposition 2.10 that the residue of the connection obtained using the above coordinates along this divisor and the new equations for the sections is given by the endomorphism of $\left.\mathbb{V}_{\bar{\lambda}}\right|_{B}$ which acts by multiplication by $c(\mu) / 2\left(\ell+h^{\vee}\right)$ on the summand $\mathbb{V}_{\bar{\lambda}^{\prime} \mu} \otimes \mathbb{V}_{\bar{\lambda}^{\prime \prime} \mu^{*}}$.

Let $D=\partial / \partial t$ and lift it to a derivation on the universal family over $U$ with trivial action in the fibre direction. To compare the connection in the new coordinates as above with the $\mathrm{KZ}$ connection we must compute the functions $a_{1}^{i}, i=1,2, \ldots, n$ occurring in Lemma 2.9. As before, we write $D=D_{\text {vert }}+D_{\text {hor }}=D_{\text {vert }}^{\prime}+D_{\text {hor }}^{\prime}$ where the ' denotes the decomposition with respect to the new coordinates.

- For the sections given $x=z_{r+1} \ldots, z_{n}, x=1$ and $1 / x=0, D_{\text {vert }}=D_{\text {vert }}^{\prime}=0$, hence $a_{1}=0$.

- For the section given by $x=0$ (this is the $n-2$ 'nd according to our numbering), we have $D_{\text {vert }}=0$ but $D_{\text {vert }}^{\prime}=-(x / t) \partial_{x}$ so $a_{1}=1 / t$.

- For the sections with equations $x=z_{i}, 1 \leq i \leq r$, by substituting $z_{i}=t w_{i}$ we see that $D_{\text {vert }}=-w_{i} \partial_{x}$ whereas $\left(\partial_{t}+(x / t) \partial_{x}\right)\left((t / x)-w_{i}^{-1}\right)=0$ so $D_{\text {vert }}^{\prime}=-(x / t) \partial_{x}=$ $-\left(\frac{x-z_{i}}{t}+w_{i}\right) \partial_{x}$. It follows that $a_{1}=1 / t$.

Adding up all the terms, we see that the residue of the $\mathrm{KZ}$ connection along this divisor is the endomorphism of $\left.\left.\mathbb{V}\right|_{\bar{\lambda}}\right|_{B}$ which acts by multiplication by

$$
\frac{c(\mu)-c\left(\lambda_{n-2}\right)-\sum_{i \in S} c\left(\lambda_{i}\right)}{2\left(\ell+h^{\vee}\right)}
$$

on the summand $\mathbb{V}_{\bar{\lambda}^{\prime} \mu} \otimes \mathbb{V}_{\bar{\lambda}^{\prime \prime} \mu^{*}}$ for each $\mu \in P_{\ell}$.

3.1.2. Boundary divisors of type (2): The change of coordinates given by $z_{i} \mapsto 1-z_{i}$, $i=1,2, \ldots, n-3$ and $x \mapsto 1-x$ on $\mathrm{M}_{0, n} \times \mathbb{P}^{1}$ preserves the equations of all the sections except for $x=0$ and $x=1$ which it interchanges and $1 / x=0$ which becomes $1 /(1-x)=0$. Moreover, it sends the locus given by $\left\{z_{i}=1\right\}_{i \in S}$ to the locus given by $\left\{z_{i}=0\right\}_{i \in S}$. Since $\partial_{i}(1 / x)=\partial_{i}(1 /(1-x))=0$ for all $i$, the $\mathrm{KZ}$ connection does not change if we replace the equation $1 / x=0$ by the equation $1 /(1-x)=0$. Using (3.1) we then see that the residue of the $\mathrm{KZ}$ connection along a divisor $B$ of type (2) is the endomorphism of $\left.\mathbb{V}_{\bar{\lambda}}\right|_{B}$ which acts by multiplication by

$$
\frac{c(\mu)-c\left(\lambda_{n-1}\right)-\sum_{i \in S} c\left(\lambda_{i}\right)}{2\left(\ell+h^{\vee}\right)}
$$

on the summand $\mathbb{V}_{\bar{\lambda}^{\prime} \mu} \otimes \mathbb{V}_{\bar{\lambda}^{\prime \prime} \mu^{*}}$ for each $\mu \in P_{\ell}$.

3.1.3. Boundary divisors of type (3): The change of coordinates given by $z_{i} \mapsto 1 / z_{i}, i=$ $1,2, \ldots, n-3$ and $x \mapsto 1 / x$, preserves the sections given by $x=z_{i}, i=1,2, \ldots, n-3$ and $x=1$ and switches the sections given by $x=0$ and $1 / x=0$. Moreover, it maps the locus given by the equations $\left\{1 / z_{i}=0\right\}_{i \in S}$ to the locus given by the equations $\left\{z_{i}=0\right\}_{i \in S}$ so we would like to use the computation for type (1) boundary divisors to compute the residue. 
However, since the equations of the sections are not preserved we must again compute the change in the connection caused by the change of coordinates.

For $i \in\{1,2, \ldots, n-3\}$ set $D=\partial_{i}$. The old as well as the new equations for all the sections except for the $i^{\prime}$ th one are killed by $\partial_{i}$, so $D_{\text {vert }}=D_{\text {vert }}^{\prime}=0$ along them. For the $i$ 'th section we have $D_{\text {vert }}=-\partial_{x}$ and since $\left(\partial_{i}-\left(x^{2} / z_{i}^{2}\right) \partial_{x}\right)\left(1 / x-1 / z_{i}\right)=0$ we have $D_{\text {vert }}^{\prime}=-x^{2} / z_{i}^{2} \partial_{x}$. Since

$$
-\frac{x^{2}}{z_{i}^{2}}=-\frac{\left(x-z_{i}\right)^{2}+2 z_{i}\left(x-z_{i}\right)}{z_{i}^{2}}-1
$$

it follows that $a_{1}=2 / z_{i}$.

To compute the difference in the residue, we must compute the difference in the action of the vector field $\partial / \partial t$ in the notation of $\S 3.1 .1$. Since $\partial / \partial t=\sum_{i \in S} w_{i} \partial_{i}$ it follows from Lemma 2.9 that this is given $\frac{\sum_{i \in S} c\left(\lambda_{i}\right)}{t\left(\ell+h^{\vee}\right)}$.

It then follows from (3.1) that the residue of the $\mathrm{KZ}$ connection along this divisor is the endomorphism of $\left.\mathbb{V}_{\bar{\lambda}}\right|_{B}$ which acts by multiplication by

$$
\frac{c(\mu)-c\left(\lambda_{n}\right)-\sum_{i \in S} c\left(\lambda_{i}\right)}{2\left(\ell+h^{\vee}\right)}+\frac{\sum_{i \in S} c\left(\lambda_{i}\right)}{\ell+h^{\vee}}=\frac{c(\mu)-c\left(\lambda_{n}\right)+\sum_{i \in S} c\left(\lambda_{i}\right)}{2\left(\ell+h^{\vee}\right)}
$$

on the summand $\mathbb{V}_{\bar{\lambda}^{\prime}} \otimes \mathbb{V}_{\bar{\lambda}^{\prime \prime} \mu^{*}}$ for each $\mu \in P_{\ell}$.

3.1.4. Boundary divisor of type (4): Again, for ease of notation we shall suppose $S=$ $\{1,2, \ldots, r\}$ for some $r, 2 \leq r \leq n-3$. Then an open subset of the blowup may be identified with $\mathbb{A}^{n-3}$ with coordinates $s, t, w_{3}, \ldots, w_{r}, z_{r+1}, \ldots, z_{n-3}$ so that the map to $\mathrm{M}_{0, n}$ is given by $\left(s, t, w_{3}, \ldots, w_{r}, z_{r+1}, \ldots, z_{n-3}\right) \mapsto\left(s, s+t, s+t w_{3}, \ldots, s+t w_{r}, z_{r+1}, \ldots, z_{n-3}\right)$ and the exceptional divisor $B$ is given by $t=0$. The universal family in a neighbourhood of $B$ is then given by blowing up the locus given by $t=x-s=0$, where $x$ is the coordinate on $\mathbb{P}^{1}$ as before.

Let $y=t /(x-s)$. All $n$ sections extend to sections of the universal family in a neighbourhood of the generic point of $T$ with equations given as follows, where $w_{2}:=1$ :

- $x-z_{1}=0$ is replaced by $1 / y=0$.

- $x-z_{i}=0$ is replaced by $y-w_{i}^{-1}=0$ for $2 \leq i \leq r$.

- The equations for the remaining sections are unchanged.

As in $\S 3.1 .1$ we now decompose $\partial_{t}$ as $D_{\text {vert }}+D_{\text {hor }}$ and $D_{\text {vert }}^{\prime}+D_{\text {hor }}^{\prime}$ and compare the two:

- For $x-z_{1}=0, D_{\text {vert }}=0$ and $D_{\text {vert }}^{\prime}=-\left(x-z_{1}\right) / t \partial_{x}$ so $a_{1}=1 / t$.

- For $x-z_{i}=0,2 \leq i \leq r$, using $z_{i}=s+t w_{i}$ we get $D_{\text {vert }}=-w_{i} \partial_{x}$ and $D_{\text {vert }}^{\prime}=$ $-(x-s) / t \partial_{x}=-\left[\left(x-\left(s+t w_{i}\right)\right) / t+w_{i}\right] \partial_{x}$. Thus $D_{\text {vert }}-D_{\text {vert }}^{\prime}=\left(x-z_{i}\right) / t \partial_{x}$, hence $a_{1}=1 / t$.

- For the remaining sections $a_{1}=0$.

As before, it follows from the above computations that the residue along this divisor is the endomorphism of $\left.\mathbb{V}_{\bar{\lambda}}\right|_{B}$ which acts by multiplication by

$$
\frac{c(\mu)-\sum_{i \in S} c\left(\lambda_{i}\right)}{2\left(\ell+h^{\vee}\right)}
$$

on the summand $\mathbb{V}_{\bar{\lambda}^{\prime} \mu} \otimes \mathbb{V}_{\bar{\lambda}^{\prime \prime} \mu^{*}}$ for each $\mu \in P_{\ell}$. 
3.2. Our formula for the Chern classes, aside from using the computations above also uses a result of Esnault and Verdier [4, Appendix B, Corollary] which we recall here for the reader's convenience:

Let $X$ be a smooth projective variety, $D=\cup_{i} D_{i}$ a divisor with simple normal crossings on $X, V$ a vector bundle on $X$ and $\nabla$ a connection on $U=X-D$ with logarithmic singularities.

Proposition 3.1 (Esnault, Verdier).

$$
N_{p}(V)=(-1)^{p} \sum_{\alpha_{1}+\cdots+\alpha_{s}=p}\left(\begin{array}{l}
p \\
\alpha
\end{array}\right) \operatorname{Tr}\left(\Gamma_{1}^{\alpha_{1}} \circ \cdots \circ \Gamma_{s}^{\alpha_{s}}\right)\left[D_{1}\right]^{\alpha_{1}} \cdots\left[D_{s}\right]^{\alpha_{s}}
$$

where $N_{p}$ denotes the $p$ 'th Newton polynomial in the Chern roots of $V,\left[D_{i}\right]$ denotes the class of $D_{i}$ in Hodge cohomology and $\Gamma_{i}$ is the endomorphism of $\left.V\right|_{D_{i}}$ given by the residue of $\nabla$ along $D_{i}$.

The KZ connection on $\mathbb{V}_{\bar{\lambda}}$ has logarithmic singularities, so using the computations of the residues of the $\mathrm{KZ}$ connection along the boundary divisors of $\overline{\mathrm{M}}_{0, n}$ and Proposition 3.1 we get the following (implicit) expression for all the Chern classes of $\mathbb{V}_{\bar{\lambda}}$ in Hodge cohomology or, equivalently, the rational Chow groups of $\overline{\mathrm{M}}_{0, n}$. The result is

Theorem 3.2. Let $\mathfrak{g}$ be a simple Lie algebra, $\ell \geq 0$ an integer and $\bar{\lambda}=\left(\lambda_{1}, \lambda_{2}, \ldots, \lambda_{n}\right) \in P_{\ell}{ }^{n}$. Then

$$
N_{p}\left(\mathbb{V}_{\bar{\lambda}}\right)=(-1)^{p} \sum_{\alpha_{1}+\cdots+\alpha_{s}=p}\left(\begin{array}{c}
p \\
\alpha
\end{array}\right) \operatorname{Tr}\left(\Gamma_{1}^{\alpha_{1}} \circ \cdots \circ \Gamma_{s}^{\alpha_{s}}\right)\left[B_{1}\right]^{\alpha_{1}} \cdots\left[B_{s}\right]^{\alpha_{s}}
$$

in $\mathrm{CH}^{p}\left(\overline{\mathrm{M}}_{0, n}\right)_{\mathbb{Q}}$. Here $N_{p}$ denotes the $p$ 'th Newton class, the $B_{i}, i=1, \ldots, s$, are the irreducible components of $\overline{\mathrm{M}}_{0, n} \backslash \mathrm{M}_{0, n}$ and $\Gamma_{i}$ denotes the residue of the $K Z$ connection along $B_{i}$ given by one of (3.1), (3.2), (3.3), and (3.4).

\section{Remarks 3.3.}

(1) Keel [13] has determined the Chow ring of $\overline{\mathrm{M}}_{0, n}$, so all intersections involved in (3.5) may be computed explicitly.

(2) To compute the traces one needs to know the ranks of the bundles of conformal blocks. These are given in closed form by the Verlinde formula for the classical groups and $G_{2}$ [19] or can be derived inductively from the 3-point ranks using [23, Corollary 3.5.2].

(3) The traces appearing in (3.5) are rational numbers but not, in general, integers.

Since the KZ connection depends on the choice of coordinates, so do the residues, hence also the representing cycle for $c_{1}\left(\mathbb{V}_{\bar{\lambda}}\right)=N_{1}\left(\mathbb{V}_{\bar{\lambda}}\right)$ in Theorem 3.2. However, by averaging over all choices we obtain a canonical representative: 
Corollary 3.4. Let $\mathfrak{g}$ be a simple Lie algebra, $\ell \geq 0$ an integer and $\bar{\lambda}=\left(\lambda_{1}, \lambda_{2}, \ldots, \lambda_{n}\right) \in$ $P_{\ell}^{n}$. Then

$$
\begin{array}{r}
(3.6) \quad c_{1}\left(\mathbb{V}_{\bar{\lambda}}\right)= \\
\frac{1}{2\left(\ell+h^{\vee}\right)} \sum_{i=2}^{\lfloor n / 2\rfloor} \epsilon_{i}\left\{\sum _ { \substack { A \subset \{ 1 , 2 , \ldots , n \} \\
| A | = i } } \left\{\frac{r_{\bar{\lambda}}}{(n-1)(n-2)}\left\{(n-i)(n-i-1) \sum_{a \in A} c\left(\lambda_{a}\right)+i(i-1) \sum_{a^{\prime} \in A^{c}} c\left(\lambda_{a^{\prime}}\right)\right\}\right.\right. \\
\left.\left.\quad-\left\{\sum_{\mu \in P_{\ell}} c(\mu) \cdot r_{\bar{\lambda}_{A, \mu}} \cdot r_{\bar{\lambda}_{A^{c}, \mu^{*}}}\right\}\right\} \cdot\left[D_{A, A^{c}}\right]\right\}
\end{array}
$$

in $\operatorname{Pic}\left(\overline{\mathrm{M}}_{0, n}\right)_{\mathbb{Q}}$, where $D_{A, A^{c}}$ is the irreducible boundary divisor corresponding to the partition $\{1,2, \ldots, n\}=A \cup A^{c}$ and $\epsilon_{i}=1 / 2$ if $i=n / 2$ and 1 otherwise.

Proof. The choices involved in $\S 3.1$ are the labelling of the last three points as $p_{0}, p_{1}$ and $p_{\infty}$. We consider all $n(n-1)(n-2)$ ways of choosing these labels and compute the cycle obtained by averaging the coefficients of $D_{A, A^{c}}$ for each possible choice.

We consider the four types of boundary divisors considered in $\S 3.1$ and consider the coefficient of $c\left(\lambda_{a}\right)$ for $a \in A$ coming from each of these divisor types. Let $i=|A|$.

(1) $p_{0} \in A$ and $p_{1}, p_{\infty} \in A^{c}$. There are $i(n-i)(n-i-1)$ such cases and from (3.1) each one of these gives a contribution of -1 , for a total contribution of $-i(n-i)(n-i-1)$.

(2) $p_{1} \in A$ and $p_{0}, p_{\infty} \in A^{c}$. There are again $i(n-i)(n-i-1)$ such cases and from (3.2) each one of these gives a contribution of -1 , for a total contribution of $-i(n-i)(n-$ $i-1)$.

(3) $p_{\infty} \in A$ and $p_{0}, p_{1} \in A^{c}$. There are $i(n-i)(n-i-1)$ such cases. From (3.3) it follows that if $a=p_{\infty}$ then we get a contribution of -1 and otherwise we get a coefficient of 1 so the total contribution is $(i-2)(n-i)(n-i-1)$.

(4) $p_{0}, p_{1}, p_{\infty} \in A^{c}$. There are $(n-i)(n-i-1)(n-i-2)$ such cases and from (3.4) it follows that each gives a contribution of -1 for a total contribution of $-(n-i)(n-$ $i-1)(n-i-2)$.

Summing all these we get that the coefficient of $c\left(\lambda_{a}\right)$ for $a \in A$ is $-n(n-i)(n-i-1)$. By symmetry it follows that the coefficient of $c\left(\lambda_{a^{\prime}}\right)$ for $a^{\prime} \in A^{c}$ is $-n i(i-1)$. The claimed formula then follows from Theorem 3.2.

Specialising Corollary 3.4 to the case $n=4$, we get the following formula which we state here for ease of reference later:

Corollary 3.5. Let $\mathfrak{g}$ be a simple lie algebra, $\ell \geq 0$ an integer and $\bar{\lambda}=\left(\lambda_{1}, \lambda_{2}, \lambda_{3}, \lambda_{4}\right) \in P_{\ell}^{4}$. Then

$$
\begin{aligned}
& \operatorname{deg}\left(\mathbb{V}_{\bar{\lambda}}\right)=\frac{1}{2\left(\ell+h^{\vee}\right)} \times\left\{\left\{r_{\bar{\lambda}} \sum_{i=1}^{4} c\left(\lambda_{i}\right)\right\}-\right. \\
& \left\{\sum_{\lambda \in P_{\ell}} c(\lambda)\left\{\left(r_{\left(\lambda_{1}, \lambda_{2}, \lambda\right)} \cdot r_{\left(\lambda_{3}, \lambda_{4}, \lambda^{*}\right)}+r_{\left(\lambda_{1}, \lambda_{3}, \lambda\right)} \cdot r_{\left(\lambda_{2}, \lambda_{4}, \lambda^{*}\right)}+r_{\left(\lambda_{1}, \lambda_{4}, \lambda\right)} \cdot r_{\left(\lambda_{2}, \lambda_{3}, \lambda^{*}\right)}\right\}\right\}\right\} .
\end{aligned}
$$


Inserting (3.7) into Proposition 2.7, one obtains a formula for $\operatorname{deg}\left(\left.\mathbb{V}_{\bar{\lambda}}\right|_{F}\right)$ for any vital curve $F$. Since the vital curves generate $\mathrm{CH}_{1}\left(\overline{\mathrm{M}}_{0, n}\right)$ this gives a dual expression for $c_{1}\left(\mathbb{V}_{\bar{\lambda}}\right)$ which will be useful to us below. Similar expressions can in principle also be obtained for the other Chern classes.

For $2 \leq i \leq n / 2$, let $D_{i}:=\epsilon_{i} \sum_{A} D_{A, A^{c}}$ where the sum is over all $A \subset\{1,2, \ldots, n\}$ with $|A|=i$ and $D_{A, A^{c}}, \epsilon_{i}$ are as above. For $\bar{\lambda} \in P_{\ell}{ }^{n}$ and $\mu \in P_{\ell}$, let $\bar{\lambda}_{A, \mu}$ be the $i+1$-tuple $\left(\lambda_{a_{1}}, \ldots, \lambda_{a_{i}}, \mu\right)$ where $A=\left\{a_{1}, \ldots, a_{i}\right\}$.

Corollary 3.6. For the action of the symmetric group $S_{n}$ on $\overline{\mathrm{M}}_{0, n}$ by permutation of the marked points we have

$$
\begin{aligned}
\sum_{\sigma \in S_{n}} \sigma^{*}\left(c_{1}\left(\mathbb{V}_{\bar{\lambda}}\right)\right)=\frac{1}{2\left(\ell+h^{\vee}\right)} \sum_{2 \leq i \leq n / 2} i !(n-i) !\left\{\left\{\left(\left(\begin{array}{c}
n-3 \\
i-1
\end{array}\right)+\left(\begin{array}{c}
n-3 \\
n-i-1
\end{array}\right)\right) \cdot r_{\bar{\lambda}} \sum_{j=1}^{n} c\left(\lambda_{j}\right)\right\}\right. \\
\left.-\left\{\sum_{|A|=i} \sum_{\mu \in P_{\ell}} c(\mu) \cdot r_{\bar{\lambda}_{A, \mu}} \cdot r_{\bar{\lambda}_{A^{c}, \mu^{*}}}\right\}\right\} \cdot\left[D_{i}\right]
\end{aligned}
$$

Proof. This follows from Theorem 3.2 and a simple counting argument.

Remark 3.7. The set $\left\{\left[D_{i}\right]\right\}_{1 \leq i \leq n / 2}$ is a basis of $\operatorname{Pic}\left(\overline{\mathrm{M}}_{0, n}\right)_{\mathbb{Q}}^{S_{n}} \cong \operatorname{Pic}\left(\overline{\mathrm{M}}_{0, n} / S_{n}\right)_{\mathbb{Q}}$, so the RHS of (3.8) is independent of all choices. Keel and McKernan [14, Theorem 1.3] have proved that the $\left[D_{i}\right]$ generate the cone of effective divisors of $\overline{\mathrm{M}}_{0, n} / S_{n}$; since $c_{1}\left(\mathbb{V}_{\bar{\lambda}}\right)$ is always nef, it follows from op. cit. that the coefficients of $\left[D_{i}\right]$ in (3.8) are all positive or all 0 . This is not the case for the coefficients of $\left[D_{A, A^{c}}\right]$ in Corollary 3.4 .

$$
\text { 4. } g=0, \mathfrak{g}=\mathfrak{s l}_{2}
$$

In this section we consider the case $\mathfrak{g}=\mathfrak{s l}_{2}$. We identify $P$ with $\mathbb{Z}$ so that $P_{+}$is identified with $\mathbb{Z}_{\geq 0}$. Then for any $\lambda \in P_{+}, c(\lambda)=\lambda^{2} / 2+\lambda$. Furthermore, $h^{\vee}=2$.

The lemma below follows from [23, Corollary 3.5.2] by using elementary facts about the representation theory of $\mathfrak{s l}_{2}$.

\section{Lemma 4.1.}

(1) For any $\ell$ and $\bar{\lambda} \in P_{\ell}^{n}, r_{\bar{\lambda}}=0$ if $\sum_{i} \lambda_{i}$ is odd, and $\mathbb{V}_{\bar{\lambda}}$ is a trivial bundle, hence has trivial determinant, if $\sum_{i} \lambda_{i} \leq 2 \ell$.

(2) For $\bar{\lambda}=\left(\lambda_{1}, \lambda_{2}, \lambda_{3}\right)$, with $\lambda_{1} \leq \lambda_{2} \leq \lambda_{3}$ and $\sum_{i} \lambda_{i}$ even we have

$$
r_{\bar{\lambda}}= \begin{cases}1 & \text { if } \lambda_{3} \leq \lambda_{1}+\lambda_{2} \text { and } \sum_{i} \lambda_{i} \leq 2 \ell \\ 0 & \text { otherwise. }\end{cases}
$$

4.1. The case $n=4$.

Proposition 4.2. Suppose $\bar{\lambda}=\left(\lambda_{1}, \lambda_{2}, \lambda_{3}, \lambda_{4}\right) \in P_{\ell}^{4}$ with $\lambda_{1} \leq \lambda_{2} \leq \lambda_{3} \leq \lambda_{4}$ and $2 s:=$ $\sum_{i} \lambda_{i}$ even. Then

$$
\operatorname{deg}\left(\mathbb{D}_{\bar{\lambda}}\right)= \begin{cases}\max \left\{0,\left(\ell+1-\lambda_{4}\right)(s-\ell)\right\} & \text { if } \lambda_{1}+\lambda_{4} \geq \lambda_{2}+\lambda_{3} \\ \max \left\{0,\left(\ell+1+\lambda_{1}-s\right)(s-\ell)\right\} & \text { if } \lambda_{1}+\lambda_{4} \leq \lambda_{2}+\lambda_{3}\end{cases}
$$


Proof. We will use descending induction on the level $\ell$. For $\ell \geq s$ the degree is 0 by Lemma 4.1, so we assume the formula holds for some $\ell \leq s$ and prove it for $\ell-1$.

Consider the first case. Since $\lambda_{4} \in P_{\ell}$ we have $\lambda_{4} \leq \ell$ so we may assume that $\lambda_{4}<\ell \leq s$. In this range, using the factorisation formula and Lemma 4.1, one sees that $r_{\bar{\lambda}}$ decreases by 1 each time $\ell$ is decreased by 1 .

We now analyse the terms occuring in the formula (3.7). Since the rank decreases by 1 in going from level $\ell$ to level $\ell-1$ it follows from factorisation that for each of the terms of the type $r_{\left(\lambda_{1}, \lambda_{2}, \lambda\right)} \cdot r_{\left(\lambda_{3}, \lambda_{4}, \lambda^{*}\right)}, r_{\left(\lambda_{1}, \lambda_{3}, \lambda\right)} \cdot r_{\left(\lambda_{2}, \lambda_{4}, \lambda^{*}\right)}$ and $r_{\left(\lambda_{1}, \lambda_{4}, \lambda\right)} \cdot r_{\left(\lambda_{2}, \lambda_{3}, \lambda^{*}\right)}$ there is exactly one $\lambda$ which contributes a non-zero term (which is actually just 1 for $\mathfrak{s l}_{2}$ ) at level $\ell$ but gives 0 at level $\ell-1$.

Suppose this $\lambda=\ell$, so $\lambda \notin P_{\ell-1}$. Considering the first type and using Lemma 4.1 (note that $\lambda=\lambda^{*}$ here) we see that we must have $\ell \leq \lambda_{1}+\lambda_{2}$ and $\ell+\lambda_{3}+\lambda_{4} \leq 2 \ell$. This implies that $\lambda_{i}=\ell / 2$ for all $i$. The second type leads to the same conclusion whereas the third gives $\ell=\lambda_{2}+\lambda_{3}=\lambda_{1}+\lambda_{4}$.

If $\lambda<\ell$, so $\lambda \in P_{\ell-1}$, it follows from Lemma 4.1 and the inequalities among the $\lambda_{i}$ that for the term of the first type we must have $\lambda_{3}+\lambda_{4}+\lambda=2 \ell$, so $\lambda=2 \ell-\left(\lambda_{3}+\lambda_{4}\right)$. Similarly, we see that we must have $\lambda=2 \ell-\left(\lambda_{2}+\lambda_{4}\right)$ for the second type, and $\lambda=2 \ell-\left(\lambda_{1}+\lambda_{4}\right)$ for the third.

Note that the formulas of the previous paragraph specialise to those of the one before that if $\lambda=\ell$.

Using the induction hypothesis, we thus see that the degree for level $\ell-1$ is given by

$$
\begin{aligned}
& 2(\ell+1) \operatorname{deg}\left(\mathbb{D}_{\bar{\lambda}}\right)=2(\ell+2)\left(\ell+1-\lambda_{4}\right)(s-\ell) \\
& \quad+c\left(2 \ell-\left(\lambda_{3}+\lambda_{4}\right)\right)+c\left(2 \ell-\left(\lambda_{2}+\lambda_{4}\right)\right)+c\left(2 \ell-\left(\lambda_{1}+\lambda_{4}\right)\right)-\sum_{i=1}^{4} c\left(\lambda_{i}\right) .
\end{aligned}
$$

Since $c(\lambda)=\lambda^{2} / 2+\lambda$ for $\mathfrak{s l}_{2}$, setting $2 s^{\prime}=\sum_{i=1}^{4} \lambda_{i}^{2}$ we see that the RHS of (4.1) is equal to

$$
\begin{aligned}
& 2(\ell+2)\left(\ell+1-\lambda_{4}\right)(s-\ell)+\left(6 \ell^{2}-4 \ell s-4 \ell \lambda_{4}+s^{\prime}+2 \lambda_{4} s\right)+\left(6 \ell-2 s-2 \lambda_{4}\right)-\left(s^{\prime}+2 s\right) \\
& =2(\ell+2)\left(\ell+1-\lambda_{4}\right)(s-\ell)+6 \ell^{2}-4 \ell s-4 \ell \lambda_{4}+2 \lambda_{4} s+6 \ell-4 s-2 \lambda_{4} \\
& =2\left[\left(\ell^{2}+\ell-\ell \lambda_{4}+2 \ell+2-2 \lambda_{4}\right)-2 \ell+\lambda_{4}-2\right] s \\
& \left.+2\left[\left(\ell^{2}+\ell-\ell \lambda_{4}+2 \ell+2-2 \lambda_{4}\right)-3 \ell+2 \lambda_{4}-3\right)\right](-\ell)-4 \lambda_{4} \\
& =2\left[(\ell+1)\left(\ell-\lambda_{4}\right)\right] s+2\left[(\ell+1)\left(\ell-\lambda_{4}\right)+\left(-\ell+\lambda_{4}-1\right)\right](-\ell)-4 \lambda_{4} \\
& =2(\ell+1)\left(\ell-\lambda_{4}\right)(s-\ell+1)
\end{aligned}
$$

Dividing by $2(\ell+1)$ we get the formula of the proposition for level $\ell-1$.

The second case is proved in an entirely analogous way so we omit the details.

4.2. The case $\ell=1$. If $\ell=1, P_{1}=\{0,1\}$ so there are $2^{n}$ bundles of conformal blocks on $\overline{\mathrm{M}}_{0, n}$. However, of these the ones corresponding to an odd number of 1 's are 0 by Lemma 4.1 and the ones with two 1's are trivial bundles of rank 1. Thus the maximal number of non-trivial determinants that one can possibly get is $2^{n-1}-\left(\begin{array}{l}n \\ 2\end{array}\right)-1$, which is the same as the rank of $\operatorname{Pic}\left(\overline{\mathrm{M}}_{0, n}\right)$. In fact, one has the following

Theorem 4.3. For any $n \geq 4$, the set of non-trivial determinants of conformal blocks of level $\ell=1$ for $\mathfrak{s l}_{2}$ form a basis of $\operatorname{Pic}\left(\overline{\mathrm{M}}_{0, n}\right)_{\mathbb{Q}}$. 
Proof. We use induction on $n$.

Suppose $n=4$. Then the only possibly non-trivial determinant is for $\bar{\lambda}=(1,1,1,1)$. From Proposition 4.2 we get that $\operatorname{deg}\left(\mathbb{D}_{\bar{\lambda}}\right)=1$, proving the first step of the induction.

Now suppose $n>4$. Let $Q$ be the quotient of $\operatorname{Pic}\left(\overline{\mathrm{M}}_{0, n}\right)_{\mathbb{Q}}$ by the subspace $P^{\prime}$ generated by $f_{i}^{*}\left(\operatorname{Pic}\left(\overline{\mathrm{M}}_{0, n-1}\right)_{\mathbb{Q}}\right), i=1,2, \ldots, n$, where the $f_{i}$ are forgetting maps as before.

Recall from [13] that for any $n \geq 4, \operatorname{Pic}\left(\overline{\mathrm{M}}_{0, n}\right)$ is generated by the classes of the boundary divisors which are parametrised by partitions $\{1, \ldots, n\}=A \cup B$ with $|A|,|B| \geq 2$. We denote the corresponding divisor by $D_{A, B}$, so we have $D_{A, B}=D_{B, A}$. For a partition $\{1,2, \ldots, \widehat{i}, \ldots, n\}=A^{\prime} \sqcup B^{\prime}$ corresponding to the boundary divisor $D_{A^{\prime}, B^{\prime}}$ on $\overline{\mathrm{M}}_{0, n-1}$ (with points labelled by elements of $\{1, \ldots, \widehat{i}, \ldots, n\})$, we have

$$
f_{i}^{*}\left(D_{A^{\prime}, B^{\prime}}\right)=D_{A^{\prime} \cup\{i\}, B^{\prime}}+D_{A^{\prime}, B^{\prime} \cup\{i\}} .
$$

Let $A=\{1,2, \ldots, r\}$ and $B=\{r+1, r+2, \ldots, n\}$ with $2 \leq r \leq n / 2$. By switching elements of $A$ and the first $r$ elements of $B$ in pairs using equation (4.2) we get

$$
D_{\{1,2, \ldots, r\},\{r+1, r+1, \ldots, n\}}=D_{\{r+1, r+2, \ldots, 2 r\},\{1,2, \ldots, r, 2 r+1, \ldots, n\}}
$$

in $Q$. Then moving $2 r+1,2 r+2, \ldots, n$ to the first set in the new partition and using the same equation we get

$$
D_{\{r+1, r+2, \ldots, 2 r\},\{1,2, \ldots, r, 2 r+1, \ldots, n\}}=(-1)^{n-2 r} D_{\{r+1, r+1, \ldots, n\},\{1,2, \ldots, r\}}=(-1)^{n} D_{\{1,2, \ldots, r\},\{r+1, r+1, \ldots, n\}}
$$

in $Q$. If $n$ is odd, it follows that $D_{A, B}=0$ in $Q$. Since the symmetric group $S_{n}$ acts on $Q$, it follows that $Q=0$ in this case. If $n$ is even, a similar argument shows that $Q$ has rank 1 , generated by $D_{\{1,2, \ldots, n / 2\},\{n / 2+1, n / 2+2, \ldots, n\}}$.

As we observed earlier, if some $\lambda_{i}=0$ then the bundle of conformal blocks is pulled back via $f_{i}$, hence its determinant is in $P^{\prime}$. To complete the proof it remains to show that $\mathbb{D}_{(1,1, \ldots, 1)}$ generates $Q$ if $n$ is even.

Suppose not, so $\mathbb{D}_{(1,1, \ldots, 1)}$ lies in $P^{\prime}$. For even $r, 4 \leq r \leq n$, let $\mathbb{D}_{r}$ be the sum of all the determinants of conformal blocks of level 1 with $r$ of the $\lambda_{i}$ equal to 1 . Since $\mathbb{D}_{n}$ is preserved by the action of $S_{n}$, it follows by averaging that we must have a linear relation

$$
\sum_{i=2}^{n / 2} a_{i} \mathbb{D}_{2 i}=0
$$

with $a_{i} \in \mathbb{Q}$.

Let $\iota: \overline{\mathrm{M}}_{0, n-1} \rightarrow \overline{\mathrm{M}}_{0, n}$ be the morphism corresponding to attaching a 3-pointed $\mathbb{P}^{1}$ to the last marked point. Let $\bar{\lambda}^{\prime}=\left(\lambda_{1}, \lambda_{2}, \ldots, \lambda_{n-2}, 0\right)$ with $\lambda_{i} \in\{0,1\}$ and $\sum_{i} \lambda_{i}=2 r \geq 4$. The coefficient of $\mathbb{D}_{\bar{\lambda}^{\prime}}$ in $\iota^{*}\left(\sum_{i=2}^{n / 2} a_{i} \mathbb{D}_{2 i}\right)$, which is well defined by induction, must be zero. By the factorisation formula this coefficient is equal to $a_{i}+a_{i+1}$ so we must have

$$
a_{i}+a_{i+1}=0,2 \leq i \leq n / 2-1 .
$$

Now let $F$ be the vital curve in $\overline{\mathrm{M}}_{0, n}$ corresponding to the partition $\{1,2, \ldots, n\}=\{1\} \cup$ $\{2\} \cup\{3\} \cup\{4,5, \ldots, n\}$. Using the computation in the $n=4$ case and Proposition 2.7 we see that for any $\bar{\lambda}=\left(\lambda_{1}, \lambda_{2}, \ldots, \lambda_{n}\right)$ with $\sum_{i} \lambda_{i}=r$ even, $4 \leq r \leq n$

$$
\mathbb{D}_{\bar{\lambda}} \cdot F= \begin{cases}1 & \text { if } \lambda_{1}=\lambda_{2}=\lambda_{3}=1 \\ 0 & \text { otherwise }\end{cases}
$$


It follows that $\mathbb{D}_{r} \cdot F=\left(\begin{array}{l}n-3 \\ r-3\end{array}\right)$ for $r$ even, $4 \leq r \leq n$. Since $\sum_{i=2}^{n / 2} a_{i} \mathbb{D}_{2 i} \cdot F=0$, by putting together the two relations obtained so far we get

$$
f(n):=\left(\begin{array}{l}
n-3 \\
n-3
\end{array}\right)-\left(\begin{array}{l}
n-3 \\
n-5
\end{array}\right)+\left(\begin{array}{l}
n-3 \\
n-7
\end{array}\right) \cdots+(-1)^{n / 2}\left(\begin{array}{c}
n-3 \\
1
\end{array}\right)=0 .
$$

However, by the binomial theorem, $2 f(n)=(1+\sqrt{-1})^{n}+(1-\sqrt{-1})^{n}$, which is clearly non-zero. This contradiction completes the proof of the theorem.

Remark 4.4. The basis of Theorem 4.3 has several nice properties which are clear from the construction: All the elements are nef line bundles and the basis is preserved by the action of the symmetric group. In fact, the basis is compatible with all natural morphisms among the $\overline{\mathrm{M}}_{0, n}$ 's including the forgetting and gluing morphisms. However, even though our basis is contained in $\operatorname{Pic}\left(\overline{\mathrm{M}}_{0, n}\right)$, it does not form an integral basis for $n>4$ as may be seen by explicit computation.

4.3. The critical level and GIT quotients. For $\bar{\lambda}=\left(\lambda_{1}, \ldots, \lambda_{n}\right)$ we call $\ell=\left(\sum_{i} \lambda_{i} / 2\right)-1$ the critical level associate to $\bar{\lambda}$. This is the largest possible value for the level so that the bundle $\mathbb{V}_{\bar{\lambda}}$ is not trivial. In this section we identify the line bundles $\mathbb{D}_{\bar{\lambda}}$ for the critical level and with all $\lambda_{i}>0$ with pullbacks of ample line bundles on the GIT quotients $\left(\mathbb{P}^{1}\right)^{n} / / S L_{2}$, where the polarisation on $\left(\mathbb{P}^{1}\right)^{n}$ is given by the line bundle $\mathcal{O}\left(\lambda_{1}\right) \otimes \cdots \otimes \mathcal{O}\left(\lambda_{n}\right)$. We will do this by comparing the degrees of the $\mathbb{D}_{\bar{\lambda}}$ on vital curves with the degrees of the GIT bundles for which a formula has been given by Alexeev-Swinarski [2].

For $\bar{\lambda}$, with none of the $\lambda_{i}=0$, let $p_{\bar{\lambda}}: \overline{\mathrm{M}}_{0, n} \rightarrow\left(\mathbb{P}^{1}\right)^{n} / / S L_{2}$ denote the morphism constructed by Kapranov [12], where the GIT quotient is constructed using the polarisation corresponding to $\bar{\lambda}$.

Theorem 4.5. For the critical level $\ell=\sum_{i} \lambda_{i} / 2-1, \mathbb{D}_{\bar{\lambda}}$ is a multiple of the pullback by $p_{\bar{\lambda}}$ of the canonical ample line bundle on $\left(\mathbb{P}^{1}\right)^{n} / / S L_{2}$. Furthermore, $\mathbb{V}_{\bar{\lambda}}$ is the pullback of a vector bundle on $\left(\mathbb{P}^{1}\right)^{n} / / S L_{2}$.

Proof. We first show that the degree of $\mathbb{D}_{\bar{\lambda}}$ on any vital curve $F$ is a fixed multiple of the degree of the GIT bundle on this curve. This suffices for the first part since $\operatorname{Pic}\left(\overline{\mathrm{M}}_{0, n}\right)$ is finitely generated and torsion free.

Let $F$ correspond to the partition $\{1, \ldots, n\}=\sqcup_{j=1}^{4} N_{j}$. For $j=1, \ldots, 4$, let $\nu_{j}=$ $\sum_{k \in N_{j}} \lambda_{k}$. Let $\nu_{\text {max }}=\max _{j}\left\{\nu_{j}\right\}$ and $\nu_{\text {min }}=\min _{j}\left\{\nu_{j}\right\}$. From Proposition 2.7 we have

$$
\left.\operatorname{deg}\left(\mathbb{D}_{\bar{\lambda}}\right)\right|_{F}=\sum_{\bar{\mu} \in P_{\ell}^{4}} \operatorname{deg}\left(\mathbb{V}_{\bar{\mu}}\right) \prod_{j=1}^{4} r_{\bar{\lambda}_{\mu_{j}^{*}}} .
$$

Note that $\mu_{j}=\mu_{j}^{*}$ since $\mathfrak{g}=\mathfrak{s l}_{2}$. To get a non-zero summand, each of its factors must be nonzero. Considering the ranks, we see from Lemma 4.1 that this implies that for each non-zero summand we must have $\mu_{j} \leq \nu_{j}$ for all $k$. Now considering the term $\operatorname{deg}\left(\mathbb{V}_{\bar{\mu}}\right)$ and applying Lemma 4.1 again, we see that we must have $\sum_{j} \mu_{j} \geq 2 \ell+2$. Since $2 \ell=\sum_{i} \lambda_{i}-2=\sum_{j} \nu_{j}-2$, it follows that all the inequalities must be equalities. Thus there is only one possibly non-zero summand corresponding to $\mu_{j}=\nu_{j}, j=1, \ldots, 4$. In this summand, since $\ell \geq 2 \nu_{j}$ for all $j$, by applying the factorisation formula one sees that $r_{\bar{\lambda}^{j}}=1$ for all $j$. 
It follows from Proposition 4.2 that

$$
\left.\operatorname{deg}\left(\mathbb{D}_{\bar{\lambda}}\right)\right|_{F}= \begin{cases}0 & \text { if } \nu_{\max } \geq \ell+1 \\ \ell+1-\nu_{\max } & \text { if } \nu_{\max } \leq \ell+1 \text { and } \nu_{\max }+\nu_{\min } \geq \ell+1 \\ \nu_{\min } & \text { if } \nu_{\max } \leq \ell+1 \text { and } \nu_{\max }+\nu_{\min } \leq \ell+1\end{cases}
$$

This is exactly the same, upto scaling, as the formula of Alexeev-Swinarski [2, Lemma 2.2]. Since the scaling factor is independent of the specific vital curve $F$, the statement about $\mathbb{D}_{\bar{\lambda}}$ follows.

Since $\mathbb{V}_{\bar{\lambda}}$ is generated by its global sections (Lemma 2.5), there exists a morphism $f_{\bar{\lambda}}$ : $\overline{\mathrm{M}}_{0, n} \rightarrow \mathrm{Gr}_{\bar{\lambda}}$, where $\mathrm{Gr}_{\bar{\lambda}}$ is a grassmannian, such that $\mathbb{V}_{\bar{\lambda}}$ is isomorphic to $f_{\bar{\lambda}}^{*}$ of the tautological vector bundle on $\mathrm{Gr}_{\bar{\lambda}}$. Since $\mathbb{D}_{\bar{\lambda}}$ is the determinant of $\mathbb{V}_{\bar{\lambda}}$ and the GIT quotient $\left(\mathbb{P}^{1}\right)^{n} / / S L_{2}$ is normal, it follows that we have $f_{\bar{\lambda}}=q_{\bar{\lambda}} \circ p_{\bar{\lambda}}$ for some morphism $q_{\bar{\lambda}}:\left(\mathbb{P}^{1}\right)^{n} / / S L_{2} \rightarrow \operatorname{Gr}_{\bar{\lambda}}$. Thus $\mathbb{V}_{\bar{\lambda}}$ is $p_{\bar{\lambda}}^{*}$ of $q_{\bar{\lambda}}^{*}$ of the tautological vector bundle.

4.4. Relationship with the moduli spaces of weighted pointed curves. For any $g \geq$ 0 , Hassett [11] has constructed moduli spaces of weighted pointed stable curves. For $g=0$, the spaces $\overline{\mathrm{M}}_{0, \mathcal{A}}$ depend on a choice of weight data $\mathcal{A}=\left(a_{1}, a_{2}, \ldots, a_{n}\right)$ satisfying $0<a_{i} \leq 1$ for all $i$ and $\sum_{i} a_{i}>2$. There are canonical birational morphisms $p_{\mathcal{A}}: \overline{\mathrm{M}}_{0, n} \rightarrow \overline{\mathrm{M}}_{0, \mathcal{A}}$.

Lemma 4.6. The morphsisms $p_{\mathcal{A}}$ are compositions of extremal contractions - in fact smooth blowdowns - corresponding to images of classes of vital curves.

The following proof was communicated to us by Valery Alexeev.

Proof. For any $S \subset\{1,2, \ldots, n\}$ such that $2<|S|<n-2$ Hassett defines an associated wall in the space $\mathcal{D}_{0, n}$ of allowable weight data given by the equation $\sum_{i \in S} a_{i}=1$. The set of all such walls induces a decompostion of $\mathcal{D}_{0, n}$ called the coarse chamber decomposition.

For each $i$ let $\epsilon_{i}$ be such that $0<\epsilon_{i}<<1$, let $a_{i}^{\prime}=a_{i}-\epsilon_{i}$ and $\mathcal{A}^{\prime}=\left(a_{1}^{\prime}, a_{2}^{\prime}, \ldots, a_{n}^{\prime}\right)$. Then there is a natural morphism $\rho_{\mathcal{A}, \mathcal{A}^{\prime}}: \overline{\mathrm{M}}_{0, \mathcal{A}} \rightarrow \overline{\mathrm{M}}_{0, \mathcal{A}^{\prime}}$ such that $\rho_{\mathcal{A}, \mathcal{A}^{\prime}} \circ p_{\mathcal{A}}=p_{\mathcal{A}^{\prime}}$ which is an isomorphism if the $\epsilon_{i}$ are sufficiently small. Thus we may assume that the $a_{i}$ 's are general, i.e., the line segment joining $\left(a_{1}, a_{2}, \ldots, a_{n}\right)$ and $(1,1 \ldots, 1)$ intersects the walls of the coarse chamber decomposition in only codimension one faces. It therefore suffices to show that each such crossing corresponds to the smooth blowdown associated to the image of a vital curve. This follows from Proposition 4.5 of [11], the vital curve may be taken to correspond to a partition $\{1,2, \ldots, n\}=\sqcup_{j=1}^{4} N_{j}$ with $S=\cup_{j=1}^{3} N_{j}$.

Given a level $\ell$ less than the critical level, i.e., $\ell<\sum_{i} \lambda_{i} / 2-1$, and an allowable tuple of weights $\bar{\lambda}$, we let $a_{i}=\lambda_{i} /(\ell+1)$ and $\mathcal{A}_{\bar{\lambda}}=\left(a_{1}, a_{2}, \ldots, a_{n}\right)$. If all $\lambda_{i}>0$, then we have $0<$ $a_{i} \leq 1$ for all $i$ and $\sum_{i} a_{i}>2$, so $\mathcal{A}_{\bar{\lambda}}$ can be taken to be weight data in the sense of Hassett. We therefore have a moduli space $\overline{\mathrm{M}}_{0, \mathcal{A}_{\bar{\lambda}}}$ and a birational morphism $p_{\mathcal{A}_{\bar{\lambda}}}: \overline{\mathrm{M}}_{0, n} \rightarrow \overline{\mathrm{M}}_{0, \mathcal{A}_{\bar{\lambda}}}$ as above.

For non-critical levels, the bundles $\mathbb{D}_{\bar{\lambda}}$ are often ample, so are not pulled back from the GIT quotients. However, we have

Proposition 4.7. If all $\lambda_{i}>0$ then $\mathbb{V}_{\bar{\lambda}}$ is the pullback by $p_{\mathcal{A}_{\bar{\lambda}}}$ of a vector bundle on $\overline{\mathrm{M}}_{0, \mathcal{A}_{\bar{\lambda}}}$.

Proof. We first show that $\mathbb{D}_{\bar{\lambda}}$ is the pullback by $p_{\mathcal{A}_{\bar{\lambda}}}$ of a line bundle on $\overline{\mathrm{M}}_{0, \mathcal{A}_{\bar{\lambda}}}$. By Lemma 4.6 it suffices to show that $\left.\operatorname{deg}\left(\mathbb{D}_{\bar{\lambda}}\right)\right|_{F}=0$ for any vital curvve $F$ contracted by $p_{\mathcal{A}_{\bar{\lambda}}}$. Let $F$ corresponds to the partition $\{1,2 \ldots, n\}=\sqcup_{j=1}^{4} N_{j}$ and let $b_{j}=\sum_{k \in N_{j}} a_{k}$. The morphism 
$p_{\mathcal{A}_{\bar{\lambda}}}$ collapses $F$ if and only if $\left(\sum_{l} b_{l}\right)-b_{j}<1$ for some $j \in\{1,2,3,4\}$. Equivalently, setting $\nu_{j}=\sum_{k \in N_{j}} \lambda_{k}$, if and only if

$$
\sum_{j} \nu_{j}-\nu_{j^{\prime}} \leq \ell \text { for some } j^{\prime} \in\{1,2,3,4\}
$$

We now apply Proposition 2.7. If a tuple $\bar{\mu} \in P_{\ell}^{4}$ is to contribute a non-zero summand, all the ranks have to be non-zero so we must have $\mu_{j} \leq \nu_{j}$ for all $j=1, \ldots, 4$. Equation (4.4) then implies that

$$
\sum_{j} \mu_{j}-\mu_{j^{\prime}} \leq \ell \text { for some } j^{\prime} \in\{1,2,3,4\}
$$

Since $\mu_{j^{\prime}} \leq \ell$ as well, we get $\sum_{j} \mu_{j} \leq 2 \ell$. It follows from Lemma 4.1 that $\operatorname{deg}\left(\mathbb{D}_{\bar{\mu}}\right)=0$.

We conclude that all the summands in the formula for $\operatorname{deg}\left(\left.\mathbb{D}_{\bar{\lambda}}\right|_{F}\right)$ are 0 , hence $\operatorname{deg}\left(\left.\mathbb{D}_{\bar{\lambda}}\right|_{F}\right)=$ 0 .

The statement about $\mathbb{V}_{\bar{\lambda}}$ now follows in the same way as the corresponding statement in Theorem 4.5 since $\overline{\mathrm{M}}_{0, \mathcal{A}_{\bar{\lambda}}}$ is smooth, hence also normal.

Remark 4.8. It is not true in general that $\mathbb{D}_{\bar{\lambda}}$ is the pullback by $p_{\mathcal{A}_{\bar{\lambda}}}$ of an ample line bundle on $\overline{\mathrm{M}}_{0, \mathcal{A}_{\bar{\lambda}}}$ : Let $n=6, \ell=1$ and $\bar{\lambda}=(1,1,1,1,1,1)$. In this case $\mathbb{D}_{\bar{\lambda}}$ has degree 0 on any vital curve corresponding to the partition $6=1+1+2+2$ and the correponding morphism is the well known birational morphism from $\overline{\mathrm{M}}_{0,6}$ to the Igusa quartic. However, the morphism $p_{\mathcal{A}_{\bar{\lambda}}}: \overline{\mathrm{M}}_{0,6} \rightarrow \overline{\mathrm{M}}_{0, \mathcal{A}_{\bar{\lambda}}}$ is an isomorphism since all $a_{i}=1 / 2$.

$$
\text { 5. } g=0 \text {, ARBITRARY } \mathfrak{g} \text { AND } \ell=1
$$

For an aribtrary simple Lie algebra $\mathfrak{g}$ we are not able to say much about the Chern classes of conformal blocks for arbitrary levels $\ell$. However, in the simplest non-trivial case of level 1 the formulae simplify considerably and we discuss them in more detail in this section. For the simply-laced Lie algebras the sheaves $\bar{\lambda}$ have rank at most one and we get the simplest formulae in these cases.

5.1. The following is the list of level 1 representations for the simple Lie algebras. We use the notation of Bourbaki [3].

- $A_{\ell}$ : all fundamental weights; $\varpi_{i}$ is dual to $\varpi_{\ell+1-i}$.

- $B_{\ell}, \ell \geq 2: \varpi_{1}$ and $\varpi_{\ell}$, i.e., the standard representation and the spin representation; these representations are self dual.

- $C_{\ell}$ : all fundamental weights; these are all self dual.

- $D_{\ell}, \ell \geq 3: \varpi_{1}, \varpi_{\ell-1}$ and $\varpi_{\ell}$, i.e., the standard representation and both the spin representations; the first representation is self dual and the other two are self dual if $\ell$ is even and dual to each other if $\ell$ is odd.

- $E_{6}: \varpi_{1}$ and $\varpi_{6}$; these representations are dual to each other.

- $E_{7}: \varpi_{7}$; this is self dual.

- $E_{8}$ : there are no level 1 representations.

- $F_{4}: \varpi_{4}$; this is self dual.

- $G_{2}: \varpi_{1}$; this is self dual. 
5.2. Recall (see e.g. [23, Corollary 3.5.2]) that for a simple Lie algebra $\mathfrak{g}$, any level $\ell$ and $\lambda, \mu \in P_{\ell}$, we have $r_{(\lambda, \mu, 0)}=1$ if $\mu=\lambda^{*}$ and 0 otherwise. Moreover, for $\lambda_{1}, \lambda_{2}, \lambda_{3}$ of level 1 , $\mathbb{V}_{\left(\lambda_{1}, \lambda_{2}, \lambda_{3}\right)}$ is the quotient of $\left(V_{\lambda_{1}} \otimes V_{\lambda_{2}} \otimes V_{\lambda_{3}}\right)_{\mathfrak{g}}$ by the image of the subspace $V_{\lambda_{1}}^{(1)} \otimes V_{\lambda_{2}}^{(1)} \otimes V_{\lambda_{3}}^{(1)}$, where $V_{\lambda_{i}}^{(1)}$ is the $\mathfrak{s}$-submodule of $V_{\lambda_{i}}$ which is the direct sum of all the non-trivial irreducible s-submodules. Since $H_{\theta}$ has no covariants on this subspace, it follows that the image of this subspace in $\left(V_{\lambda_{1}} \otimes V_{\lambda_{2}} \otimes V_{\lambda_{3}}\right)_{\mathfrak{g}}$ is $\{0\}$. Therefore $\mathbb{V}_{\left(\lambda_{1}, \lambda_{2}, \lambda_{3}\right)}=\left(V_{\lambda_{1}} \otimes V_{\lambda_{2}} \otimes V_{\lambda_{3}}\right)_{\mathfrak{g}}$.

5.2.1. $A_{\ell}$. In this case given $\lambda_{1}, \lambda_{2}$ of level 1 , there exists a unique $\lambda_{3}$ of level 1 such that $r_{\left(\lambda_{1}, \lambda_{2}, \lambda_{3}\right)}=1$. By the factorisation formula, it follows that for $\lambda_{1}, \lambda_{2}, \ldots, \lambda_{n-1}$ of level 1 , there exists a unique $\lambda_{n}$ of level 1 such that for $\bar{\lambda}=\left(\lambda_{1}, \lambda_{2}, \ldots, \lambda_{n}\right), r_{\bar{\lambda}} \neq 0$. Moreover, it then follows that $r_{\bar{\lambda}}=1$. Because of this there is only one non-zero summand in the formula in Proposition 2.7 and so the computation of the determinants reduces to the computation of the degree for the case $n=4$.

Note that

$$
c\left(\varpi_{i}\right)=\left(\varpi_{i} \mid \varpi_{i}\right)+2\left(\varpi_{i} \mid \rho\right)=i(m-i) / m+i(m-i)=i(m-i)(m+1) / m .
$$

where $m=\ell+1$. Using this, the fact that $h^{\vee}$ for $\mathfrak{s l}_{m}$ is $m$ and formula (3.7), one easily checks the following:

Lemma 5.1. Let $\varpi_{i}, \varpi_{j}, \varpi_{k}, \varpi_{l}$ be fundamental dominant weights of $\mathfrak{s l}_{m}$ and suppose that $i \leq j \leq k \leq l$. For $\bar{\lambda}=\left(\varpi_{i}, \varpi_{j}, \varpi_{k}, \varpi_{l}\right)$, we have

$$
\operatorname{deg}\left(\mathbb{D}_{\bar{\lambda}}\right)= \begin{cases}i & \text { if } i+j+k+l=2 m \text { and } j+k \geq i+l, \\ m-l & \text { if } i+j+k+l=2 m \text { and } j+k \leq i+l, \\ 0 & \text { otherwise. }\end{cases}
$$

From this and Proposition 2.7 we immediately get the following:

Proposition 5.2. Let $\bar{\lambda}=\left(\varpi_{i_{1}}, \varpi_{i_{2}}, \ldots, \varpi_{i_{n}}\right)$ with $0 \leq i_{j}<m$ for $j=1,2, \ldots, n$, where $\varpi_{0}:=0$. Let $F$ be a vital curve in $\overline{\mathrm{M}}_{0, n}$ corresponding to a partition $\{1,2, \ldots, n\}=\sqcup_{k=1}^{4} N_{k}$. Let $\nu_{k}$ be the representative in $\{0,1, \ldots, m-1\}$ of $\sum_{j \in N_{k}} i_{j}$ modulo $m$. Let $\nu_{\max }=\max _{k}\left\{\nu_{k}\right\}$ and $\nu_{\min }=\min _{k}\left\{\nu_{k}\right\}$. Then

$$
\operatorname{deg}\left(\left.\mathbb{D}_{\bar{\lambda}}\right|_{F}\right)= \begin{cases}\nu_{\min } & \text { if } \sum_{k} \nu_{k}=2 m \text { and } \nu_{\max }+\nu_{\min } \leq m, \\ m-\nu_{\max } & \text { if } \sum_{k} \nu_{k}=2 m \text { and } \nu_{\max }+\nu_{\min } \geq m, \\ 0 & \text { otherwise. }\end{cases}
$$

Remark 5.3. Comparing this formula with the formula for the degrees for $\mathfrak{s l}_{2}$ and the critical level (4.3), we see that the critical level $\ell$ determinants for $\mathfrak{s l}_{2}$ are a special case for the level 1 determinants for $\mathfrak{s l}_{\ell+1}$. More precisely, given an $n$-tuple of non-negative integers $\left(i_{1}, i_{2}, \ldots, i_{n}\right)$ such that $\sum_{j} i_{j}$ is even, we let $\ell=\sum_{j} i_{j} / 2-1$. Then the determinant of the bundle of conformal blocks on $\overline{\mathrm{M}}_{0, n}$ associated to $\mathfrak{s l}_{2}$ with weights $\left(i_{1}, i_{2}, \ldots, i_{n}\right)$ and level $\ell$ is isomorphic to the bundle of conformal blocks (it is already of rank 1) on $\overline{\mathrm{M}}_{0, n}$ associated to $\mathfrak{s l}_{\ell+1}$ with weights $\left(\varpi_{i_{1}}, \varpi_{i_{2}}, \ldots, \varpi_{i_{n}}\right)$ and level 1 .

5.2.2. $B_{\ell}$. In this case we have $r_{\left(\varpi_{1}, \varpi_{1}, \varpi_{1}\right)}=r_{\left(\varpi_{\ell}, \varpi_{\ell}, \varpi_{\ell}\right)}=r_{\left(\varpi_{1}, \varpi_{1}, \varpi_{\ell}\right)}=0$ whereas $r_{\left(\varpi_{1}, \varpi_{\ell}, \varpi_{\ell}\right)}=$ 1. From this and the factorisation formula, one sees that for $\bar{\lambda} \in P_{1}^{n}, r_{\bar{\lambda}}=0$ unless $m$, the number of $\lambda_{i}$ equal to $\varpi_{\ell}$, is even. If $m=0$ then the number of $\varpi_{1}$ must be even and then 
$r_{\bar{\lambda}}=1$ whereas if $m>0$ then the rank is $2^{m / 2-1}$. The possible $\bar{\lambda} \in P_{1}^{4}$ with $\operatorname{deg}\left(\mathbb{V}_{\bar{\lambda}}\right)>0$ are, upto order, $\left(\varpi_{1}, \varpi_{1}, \varpi_{1}, \varpi_{1}\right),\left(\varpi_{1}, \varpi_{1}, \varpi_{\ell}, \varpi_{\ell}\right)$ and $\left(\varpi_{\ell}, \varpi_{\ell}, \varpi_{\ell}, \varpi_{\ell}\right)$.

From the tables in [3] we see that $h^{\vee}=2 \ell-1, c\left(\varpi_{1}\right)=2 \ell$ and $c\left(\varpi_{\ell}\right)=\frac{\ell(2 \ell+1)}{4}$.

5.2.3. $C_{\ell}$. In this case, the fusion for level 1 is similar to the fusion for $\mathfrak{s l}_{2}$ at level $\ell$, i.e., the bijection between $P_{1, \mathfrak{s p}_{2 \ell}}$ and $P_{\ell, \mathfrak{s l}_{2}}$ which sends $\varpi_{i}$ to $i$ preserves the 3 -point ranks. This can be seen by explicit computation using the generalised Littlewood-Richardson rule of Littelmann [15, p. 42]. By factorisation, it follows that all $n$-point ranks are preserved. For the degree in the case $n=4$, we have:

Proposition 5.4. Suppose $n=4, i \leq j \leq k \leq l$ and $2 s:=i+j+k+l$ is even. Then for any rank $\ell \geq l$ and $\bar{\lambda}=\left(\varpi_{i}, \varpi_{j}, \varpi_{k}, \varpi_{l}\right)$ we have

$$
\operatorname{deg}\left(\mathbb{D}_{\bar{\lambda}}\right)= \begin{cases}\max \{0,(\ell+1-l)(2 s-\ell-l) / 2\} & \text { if } i+l \geq j+k \text { and } \ell \leq s, \\ (s+1-l)(s-l) / 2 & \text { if } i+l \geq j+k \text { and } \ell \geq s \\ \max \{0,(\ell+1+i-s)(i-\ell+s) / 2\} & \text { if } i+k \leq j+l \text { and } \ell \leq s \\ i(i+1) / 2 & \text { if } i+k \leq j+l \text { and } \ell \geq s\end{cases}
$$

Proof. From the tables in [3], one computes that for $\mathfrak{s p}_{2 \ell}, h^{\vee}=\ell+1$ and $c_{\ell}\left(\varpi_{i}\right)=i(\ell-i / 2+1)$, where we use a subscript for the Casimir action to emphasize that it depends on $\ell$. Using this, one may prove the proposition in a similar way to the proof of Proposition 4.2 so we only describe the changes that need to be made.

Consider the first case. Since the degree is not necessarily zero for large $\ell$ we use (increasing) induction beginning with the base case $\ell=l$. It follows from factorisation that for each of the terms of the type $r_{\left(\varpi_{i}, \varpi_{j}, \lambda\right)} \cdot r_{\left(\varpi_{k}, \varpi_{l}, \lambda^{*}\right)}, r_{\left(\varpi_{i}, \varpi_{k}, \lambda\right)} \cdot r_{\left(\varpi_{j}, \varpi_{l}, \lambda^{*}\right)}$ and $r_{\left(\varpi_{i}, \varpi_{l}, \lambda\right)} \cdot r_{\left(\varpi_{j}, \varpi_{k}, \lambda^{*}\right)}$ occuring in the formula of Corollary 3.5, there is exactly one $\lambda$ which contributes a non-zero term (which is actually just 1); in particular the rank is 1 . These $\lambda$ are seen to be, in order, $\varpi_{l-k}, \varpi_{l-j}$ and $\varpi_{l-i}$. The degree is therefore given by

$$
\frac{1}{2(l+2)}\left\{c_{\ell}\left(\varpi_{i}\right)+c_{\ell}\left(\varpi_{j}\right)+c_{\ell}\left(\varpi_{k}\right)+c_{\ell}\left(\varpi_{l}\right)-\left\{c_{\ell}\left(\varpi_{l-i}\right)+c_{\ell}\left(\varpi_{l-j}\right)+c_{\ell}\left(\varpi_{l-k}\right)\right\}\right\} .
$$

Substituting in the values of the $c_{\ell}$, simple algebraic manipulations show that this is equal to $s-l$, proving the base case. As seen before for the case of $\mathfrak{s l}_{2}$, the rank increases by 1 each time the level increases by 1 until $\ell=s$, so we may prove the formula for all $\ell$ such that $l \leq \ell \leq s$ by induction as before.

We now consider the second case. It follows from the first case that the formula holds for $\ell=s$, so we use induction to prove it for larger $\ell$. For all such $\ell$, the rank is constant and all the terms in (3.7) for varying $\ell$ are the same except for the fact that the Casimir actions depend on $\ell$. More precisely, we have

$$
\operatorname{deg}_{\ell}\left(\mathbb{V}_{\bar{\lambda}}\right)=\frac{1}{2(\ell+2)}\left\{c_{\ell}\left(\varpi_{i}\right)+c_{\ell}\left(\varpi_{j}\right)+c_{\ell}\left(\varpi_{k}\right)+c_{\ell}\left(\varpi_{l}\right)-\sum_{\lambda \in P_{1}} c_{\ell}(\lambda) \cdot a_{\lambda}\right\}
$$

where $a_{\lambda} \in\{0,1,2,3\}$ is independent of $\ell$ in this range. It follows from this and the formula for $c_{\ell}$ that $\lim _{\ell \rightarrow \infty} \operatorname{deg}_{\ell}\left(\mathbb{V}_{\bar{\lambda}}\right)$ exists. Since $\operatorname{deg}_{\ell}\left(\mathbb{V}_{\bar{\lambda}}\right)$ is always an integer it follows that $\operatorname{deg}_{\ell}\left(\mathbb{V}_{\bar{\lambda}}\right)$ is constant for $\ell \gg 0$. 
The formula also shows that

$$
\begin{aligned}
& 2(\ell+2)(\ell+3)\left(\operatorname{deg}_{\ell}\left(\mathbb{V}_{\bar{\lambda}}\right)-\operatorname{deg}_{\ell+1}\left(\mathbb{V}_{\bar{\lambda}}\right)\right) \\
= & (\ell+3)\left\{c_{\ell}\left(\varpi_{i}\right)+c_{\ell}\left(\varpi_{j}\right)+c_{\ell}\left(\varpi_{k}\right)+c_{\ell}\left(\varpi_{l}\right)-\sum_{\lambda \in P_{1}} c_{\ell}(\lambda) \cdot a_{\lambda}\right\} \\
- & (\ell+2)\left\{c_{\ell+1}\left(\varpi_{i}\right)+c_{\ell+1}\left(\varpi_{j}\right)+c_{\ell+1}\left(\varpi_{k}\right)+c_{\ell+1}\left(\varpi_{l}\right)-\sum_{\lambda \in P_{1}} c_{\ell+1}(\lambda) \cdot a_{\lambda}\right\} \\
= & (\ell+3)\left\{c_{\ell}\left(\varpi_{i}\right)+c_{\ell}\left(\varpi_{j}\right)+c_{\ell}\left(\varpi_{k}\right)+c_{\ell}\left(\varpi_{l}\right)-\sum_{\lambda \in P_{1}} c_{\ell}(\lambda) \cdot a_{\lambda}\right\} \\
- & (\ell+3)\left\{c_{\ell+1}\left(\varpi_{i}\right)+c_{\ell+1}\left(\varpi_{j}\right)+c_{\ell+1}\left(\varpi_{k}\right)+c_{\ell+1}\left(\varpi_{l}\right)-\sum_{\lambda \in P_{1}} c_{\ell+1}(\lambda) \cdot a_{\lambda}\right\} \\
+ & \left\{c_{\ell+1}\left(\varpi_{i}\right)+c_{\ell+1}\left(\varpi_{j}\right)+c_{\ell+1}\left(\varpi_{k}\right)+c_{\ell+1}\left(\varpi_{l}\right)-\sum_{\lambda \in P_{1}} c_{\ell+1}(\lambda) \cdot a_{\lambda}\right\} \\
= & \left.-(\ell+3)\left\{i+j+k+l-\sum_{\lambda \in P_{1}} \lambda \cdot a_{\lambda}\right\} \quad c_{\lambda \in P_{1}} c_{\ell+1}(\lambda) \cdot a_{\lambda}\right\} . \\
+ & \left\{c_{\ell+1}\left(\varpi_{i}\right)+c_{\ell+1}\left(\varpi_{j}\right)+c_{\ell+1}\left(\varpi_{k}\right)+c_{\ell+1}\left(\varpi_{l}\right)-\sum\right.
\end{aligned}
$$

For $\ell \gg 0$, we have seen that this is 0 so $\operatorname{deg}_{\ell}\left(\mathbb{V}_{\bar{\lambda}}\right)=\left\{i+j+k+l-\sum_{\lambda \in P_{1}} \lambda \cdot a_{\lambda}\right\} / 2$ for such $\ell$. If the formula is known for level $\ell+1$ with $\ell \geq s$, then the above equations imply that it also holds for level $\ell$. This implies $\operatorname{deg}_{\ell}\left(\mathbb{V}_{\bar{\lambda}}\right)$ is constant for all $\ell \geq s$, hence is equal to $\operatorname{deg}_{s}\left(\mathbb{V}_{\bar{\lambda}}\right)$, completing the proof in this case.

The remaing two cases are derived in a very similar way so we omit the details.

Comparing Proposition 5.4 with Proposition 4.2, we see that the degree for $\mathfrak{s p}_{2 \ell}$ is always greater than or equal to the corresponding degree for $\mathfrak{s l}_{2}$. It follows that for any $n \geq 4$ and $\bar{\lambda} \in P_{1, \mathfrak{s p}_{2 \ell}}{ }^{n} \leftrightarrow P_{\ell, \mathfrak{s l}_{2}}{ }^{n}$, the line bundle $\mathbb{D}_{\bar{\lambda}}^{\mathfrak{s p}_{2 \ell}} \otimes\left(\mathbb{D}_{\bar{\lambda}}^{\mathfrak{s l}_{2}}\right)^{-1}$ has non-negative degree on every vital curve. However, we do not know if this line bundle is nef.

Remark 5.5. It seems possible that similar results might hold more generally, with conformal blocks of $\mathfrak{s p}_{2 r}$ at level $\ell$ being "more positive" than the corresponding conformal blocks of $\mathfrak{s p}_{2 \ell}$ at level $r$ if $r \geq \ell$. Here one should use the bijection between $P_{\ell, \mathfrak{s p}_{2 r}}$ and $P_{r, \mathfrak{s p}_{2 \ell}}$ given by replacing a Young tableau with its transpose. Note that this is not the same as the bijection used by Abe in [1] in his formulation of "strange duality".

5.2.4. $D_{\ell}$. Let $R \subset P$ denote the root lattice. The quotient map $P \rightarrow P / R$ identifies $P_{1}$ with $P / R$ giving the former the structure of an abelian group whose operation we denote by $\odot$. It is known, see for example [1], that for $\bar{\lambda}=\left(\lambda_{1}, \lambda_{2}, \lambda_{3}\right) \in P_{1}^{3}, r_{\bar{\lambda}}=1$ iff $\lambda_{1} \odot \lambda_{2} \odot \lambda_{3}=0$ and $r_{\bar{\lambda}}=0$ otherwise. It follows from the the factorisation formula that for general $\bar{\lambda} \in P_{1}^{n}$, $\mathbb{V}_{\bar{\lambda}}$ is non-zero iff $\bigodot_{i} \lambda_{i}=0$, in which case it is always a line bundle.

Let $F$ be a vital curve in $\overline{\mathrm{M}}_{0, n}$ corresponding to a partition $\{1,2, \ldots, n\}=\sqcup_{k=1}^{4} N_{k}$ and for each $k$, let $\nu_{k}=\bigodot_{j \in N_{k}} \lambda_{j}$ and $\bar{\nu}:=\left(\nu_{1}, \nu_{2}, \nu_{3}, \nu_{4}\right)$. Then by Proposition $2.7, \operatorname{deg}\left(\left.\mathbb{D}_{\bar{\lambda}}\right|_{F}\right)=$ $\operatorname{deg}\left(\mathbb{D}_{\bar{\nu}}\right)$. If any $\nu_{i}=0$, then $\operatorname{deg}\left(\mathbb{D}_{\bar{\nu}}\right)=0$ and for $\mathbb{D}_{\bar{\nu}}$ to be nontrivial we must also have $\sum_{k=1}^{4} \nu_{k} \in R$. Furthermore, 
- If $\ell$ is even it follows that we must have, upto ordering, $\nu_{1}=\nu_{2} \neq 0$ and $\nu_{3}=\nu_{4} \neq 0$ in order to get a non-zero degree. In this case, it then follows from Corollary 3.5 that

$$
\operatorname{deg}\left(\mathbb{D}_{\bar{\nu}}\right)= \begin{cases}\frac{\ell}{2} & \text { if all } \nu_{k}=\nu \in\left\{\varpi_{\ell-1}, \varpi_{\ell}\right\}, \\ 2 & \text { if all } \nu_{k}=\varpi_{1}, \\ \frac{\ell-2}{2} & \text { if all } \nu_{k} \in\left\{\varpi_{\ell-1}, \varpi_{\ell}\right\} \text { but not all equal, } \\ 1 & \text { otherwise. }\end{cases}
$$

- If $\ell$ is odd it follows that we must have all $\nu_{k}$ equal or, upto order, $\bar{\nu}=\left(\varpi_{1}, \varpi_{1}, \varpi_{\ell-1}, \varpi_{\ell}\right)$ or $\left(\varpi_{\ell-1}, \varpi_{\ell-1}, \varpi_{\ell}, \varpi_{\ell}\right)$. Applying Corollary 3.5 again we see that

$$
\operatorname{deg}\left(\mathbb{D}_{\bar{\nu}}\right)= \begin{cases}\frac{\ell-3}{2} & \text { if all } \nu_{k}=\nu \in\left\{\varpi_{\ell-1}, \varpi_{\ell}\right\}, \\ 2 & \text { if all } \nu_{k}=\varpi_{1}, \\ \frac{\ell-1}{2} & \text { if all } \nu_{k} \in\left\{\varpi_{\ell-1}, \varpi_{\ell}\right\} \text { but not all equal, } \\ 1 & \text { otherwise. }\end{cases}
$$

From the tables in [3] we have used that $h^{\vee}=2 \ell-2$ and one also calculates that $c\left(\varpi_{1}\right)=$ $2 \ell-1, c\left(\varpi_{\ell-1}\right)=c\left(\varpi_{\ell}\right)=\frac{\ell(2 \ell-1)}{4}$.

For $n>4$, one easily sees from the above that all the $\mathbb{D}_{\bar{\lambda}}$ lie on the boundary of the nef cone. As $\ell$ varies, keeping its parity the same, the non-trivial conformal blocks are indexed by the same data so we identify all the sets $P_{1, \mathfrak{s o}_{2 \ell}}$ for $\ell$ even with the set $P_{1, \text { even }}:=\left\{0, \varpi_{1}, \varpi_{e-1}, \varpi_{e}\right\}$ with $e$ a formal symbol and similarly for $\ell$ odd, with $e$ replaced by $o$. The set of vital curves $F$ on which the degree is 0 is preserved by this indexing but the bundles themselves are not. We will use $\ell$ as a superscript in order to specify the level.

Proposition 5.6. For any integer integer $n \geq 4$, the closed subcone of $N_{1}\left(\overline{\mathrm{M}}_{0, n}\right)_{\mathbb{R}}$ generated by $c_{1}\left(\mathbb{D} \frac{\ell}{\lambda}\right)$ for all $\bar{\lambda} \in P_{1, \text { even }}^{n}, \ell \geq 4$ and even, or $\bar{\lambda} \in P_{1, \text { odd }}^{n}, \ell \geq 3$ and odd, is a finitely generated rational polyhedral cone.

Proof. It follows from (5.1) and (5.2) and the preceding discussion that for any $\bar{\lambda} \in P_{1 \text {,even }}^{n}$ or $P_{1, \text { odd }}^{n}$, we may divide $\mathbb{D} \frac{\ell}{\lambda}$ by $\ell / 2$ and take the limit as $\ell \rightarrow \infty$ (while keeping its parity fixed) to get $\mathbb{Q}$-line bundles on $\overline{\mathrm{M}}_{0, n}$ which we denote by $\mathbb{D}_{\bar{\lambda}}^{\text {even }}$ and $\mathbb{D}_{\bar{\lambda}}^{\text {odd }}$. We have

- For $\bar{\nu} \in P_{1, \text { even }}^{4}$ such that upto ordering $\nu_{1}=\nu_{2} \neq 0$ and $\nu_{3}=\nu_{4} \neq 0$. Then

$$
\operatorname{deg}\left(\mathbb{D}_{\bar{\nu}}^{\text {even }}\right)= \begin{cases}1 & \text { if all } \nu_{k} \in\left\{\varpi_{e-1}, \varpi_{e}\right\} \\ 0 & \text { otherwise }\end{cases}
$$

- For $\bar{\nu} \in P_{1, \text { odd }}^{4}$ such that all $\nu_{k}$ are equal or, upto order, $\bar{\nu}=\left(\varpi_{1}, \varpi_{1}, \varpi_{o-1}, \varpi_{o}\right)$ or $\left(\varpi_{o-1}, \varpi_{o-1}, \varpi_{o}, \varpi_{o}\right)$. Then

$$
\operatorname{deg}\left(\mathbb{D}_{\bar{\nu}}^{\text {odd }}\right)= \begin{cases}1 & \text { if all } \nu_{k} \in\left\{\varpi_{o-1}, \varpi_{o}\right\} \\ 0 & \text { otherwise }\end{cases}
$$

It is then immediate that for $\ell \geq 4$ and even we have $\mathbb{D} \frac{\ell}{\lambda}=\mathbb{D} \frac{4}{\lambda}+\frac{\ell-4}{2} \cdot \mathbb{D} \frac{\infty}{\lambda}$ and for $\ell \geq 3$ and odd we have $\mathbb{D} \frac{\ell}{\lambda}=\mathbb{D} \frac{3}{\lambda}+\frac{\ell-3}{2} \cdot \mathbb{D} \frac{\infty}{\lambda}$. It follows that the closed cone generated by all $c_{1}\left(\mathbb{D} \frac{\ell}{\lambda}\right)$ is generated by the first Chern classes of $\mathbb{D} \frac{\ell}{\lambda}, \ell=3,4, \mathbb{D} \frac{\text { even }}{\lambda}, \mathbb{D} \frac{\text { odd }}{\lambda}$ and there are only finitely many choices for $\bar{\lambda}$ for a fixed $n$. 
Remark 5.7. We do not know whether $\mathbb{D}_{\frac{\text { even }}{\lambda}}$ and $\mathbb{D}_{\frac{\mathrm{o}}{\lambda}}^{\text {dd }}$ are semiample. Note that since their degree on any vital curve is 0 or 1 , in particular an integer, they are actually line bundles and not just $\mathbb{Q}$-line bundles. It would be interesting to have a more geometric description of these bundles.

5.2.5. $E_{6}$. In this case $^{1}, r_{(\lambda, \lambda, \lambda)}=1$ for $\lambda=\varpi_{1}$ or $\varpi_{6}$. Since the representations are not self-dual, it follows that the conformal blocks of level 1 for $E_{6}$ are the same, again upto scaling, as those for $\mathfrak{s l}_{3}$.

5.2.6. $E_{7}$. In this case, $\left(V_{\varpi_{7}} \otimes V_{\varpi_{7}} \otimes V_{\varpi_{7}}\right)_{E_{7}}=0$, so the corresponding conformal block is also trivial. It follows that for $E_{7}$ the determinants of conformal blocks of level 1 are the same, upto scaling, as those for $\mathfrak{s l}_{2}$.

5.2.7. $E_{8}$. As there are no non-trivial representations of level $\leq 1$ we do not get any nontrivial conformal blocks. In higher genus the situation is more interesting as we see in Corollary 6.2 .

5.2.8. $F_{4}$ and $G_{2}$. In both these cases the rank of the conformal blocks for $\bar{\lambda}=\left(\varpi_{4}, \varpi_{4}, \varpi_{4}\right)$ for $F_{4}$ and $\bar{\lambda}=\left(\varpi_{1}, \varpi_{1}, \varpi_{1}\right)$ for $G_{2}$ is 1 . It follows that the determinants of the conformal blocks at level 1 for both these cases are equal (upto a global scalar). Moreover, for $\bar{\lambda}=$ $\left(\varpi_{4}, \varpi_{4}, \varpi_{4}, \varpi_{4}\right)$ for $F_{4}$ and $\bar{\lambda}=\left(\varpi_{1}, \varpi_{1}, \varpi_{1} \varpi_{1}\right)$ for $G_{2}, \operatorname{deg}\left(\mathbb{D}_{\bar{\lambda}}\right)>0$.

For any $n$ and $\bar{\lambda}=(\lambda, \lambda, \ldots, \lambda), \lambda=\varpi_{4}$ or $\varpi_{1}$ as $\mathfrak{g}=F_{4}$ or $G_{2}$, it follows from factorisation and the above that $\mathbb{V}_{\bar{\lambda}}$ has rank $F i b(n-1)$, where $F i b(i)$ denotes the $i$ 'th Fibonacci number. Moreover, Proposition 2.7 implies that if a vital curve $F$ corresponds to a partition $\{1,2, \ldots, n\}=\sqcup_{k=1}^{4} N_{k}$, then $\left.\operatorname{deg}\left(\mathbb{V}_{\bar{\lambda}}\right)\right|_{F}=c \prod_{k=1}^{4} F i b\left(\left|N_{k}\right|\right)$ where $c$ is a positive constant (which can be determined). One sees that the $\mathrm{F}$-conjecture imples that $\mathbb{D}_{\bar{\lambda}}$ is ample.

\section{THE CASE $g>0$}

As we have remarked before, for general $g$ the WZW/Hitchin connection does not always lift to a flat connection on $\mathbb{V}_{g, n, \bar{\lambda}}$ restricted to $\mathrm{M}_{g, n}$, so we cannot directly apply the same method as in the $g=0$ case. However, we do get a flat connection on all vector bundles induced by representations of $G L_{r}, r=\operatorname{rank}\left(\mathbb{V}_{g, n, \bar{\lambda}}\right)$, which are trivial on the centre. The Chern classes of all such bundles may, in principle, be computed as before provided that we can compute all intersections of boundary divisors. Note that for such bundles the connection is canonical, so one does not need to account for choices of coordinates and all one needs is Proposition 2.10.

To compute the Chern classes of $\mathbb{V}_{g, n, \bar{\lambda}}$ itself, it suffices to know the Chern classes of the associated bundles as above along with $c_{1}\left(\mathbb{V}_{g, n, \bar{\lambda}}\right)$. By Remark 2.8 and Corollary 3.5 it follows that to compute $c_{1}\left(\mathbb{V}_{g, n, \bar{\lambda}}\right)$ in general it suffices to consider the case $(g, n)=(1,1)$.

6.1. The case $g=1, n=1$. For the rest of this section, contrary to our earlier notation, for $\lambda \in P_{\ell}$ we shall denote by $\mathbb{V}_{\bar{\lambda}}$ the bundle of conformal blocks $\mathbb{V}_{1,1, \lambda}$. Our main result is:

Theorem 6.1. Let $\mathfrak{g}$ be a simple Lie algebra, $\ell \geq 0$ an integer and $\lambda \in P_{\ell}$. Then

$$
\operatorname{deg}\left(\mathbb{V}_{\lambda}\right)=\frac{1}{2\left(\ell+h^{\vee}\right)} \cdot\left\{\frac{r_{\lambda}\left(c_{\lambda}+\ell \operatorname{dim}(\mathfrak{g})\right)}{12}-\sum_{\mu \in P_{\ell}} c_{\mu} r_{\left(\lambda, \mu, \mu^{*}\right)}\right\} .
$$

\footnotetext{
${ }^{1}$ This and other such computations for exceptional groups mentioned below were carried out using GAP [7].
} 
Proof. In order to apply the results of $\S 2.3$ we must make explicit choices for a smooth projective curve mapping onto $\overline{\mathrm{M}}_{1,1}$, a coordinate for the zero section over the smooth locus and a bidifferential. The particular choices do not really matter; what is important is that choices can be made so that we get a well defined connection on the pullback of $\mathbb{V}_{\bar{\lambda}}$ over the smooth locus.

For simplicity, we shall now work over the field of complex numbers $\mathbb{C}$. The formula for the degree we shall obtain will clearly hold over any field of characteristic zero.

Let $\Gamma(8)$ be the principal congruence subgroup of $S L_{2}(\mathbb{Z})$, of level 8 and $Y(8)$ the corresponding modular curve, i.e., the quotient $\mathfrak{H} / \Gamma(8)$, see e.g. [18, Appendix A, §13]. The semi-direct product $\mathbb{Z}^{2} \ltimes \Gamma(8)$ acts on on $\mathbb{C} \times \mathfrak{H}$ by

$$
\left((m, n),\left(\begin{array}{ll}
a & b \\
c & d
\end{array}\right)\right):(z, \tau) \mapsto\left(\frac{z+m \tau+n}{c \tau+d}, \frac{a \tau+b}{c \tau+d}\right)
$$

where $z$ (resp. $\tau)$ is the usual coordinate on $\mathbb{C}($ resp. $\mathfrak{H})$. The quotient is the universal family of elliptic curves with full level 8 structure over $Y(8)$. It extends to a semi-stable family over the smooth compactification $X(8)$ of $Y(8)$ thus giving rise to a surjective morphism $X(8) \rightarrow \overline{\mathrm{M}}_{1,1}$.

Set $q=\exp (\pi i \tau)$ and $w=\exp (\pi i z)$, and consider the functions $f_{1}$ and $f_{2}$ given by

$$
f_{1}:=\vartheta_{11}(2 z, \tau)=\vartheta_{11}\left(w^{2}, q\right)=\sum_{m \in \mathbb{Z}}(-1)^{m} q^{\left(m+\frac{1}{2}\right)^{2}} w^{4 m+2}
$$

and

$$
f_{2}:=\vartheta_{00}(2 z, \tau)=\vartheta_{00}\left(w^{2}, q\right)=\sum_{m \in \mathbb{Z}} q^{m^{2}} w^{4 m} .
$$

The functions $\vartheta_{00}$ and $\vartheta_{11}$ are two of the classical Jacobi theta functions as defined, for example, in [17].

It follows from the transformation formulae in $\left[17\right.$, p. 55] that $f_{1} / f_{2}$ descends to a well defined rational function on the universal family over $Y(8)$ and gives a coordinate for the zero section. This is the coordinate we shall use.

For an elliptic curve $E=\mathbb{C} / \Lambda$ there is a natural bidifferential on $E \times E$ given by

$$
\omega:=\wp_{\Lambda}(x-y) d x d y
$$

where $\wp_{\Lambda}$ denotes the Weirstrass $\wp$ function associated to the lattice $\Lambda$. One checks that this is well defined, i.e. does not depend on the presentation of $E$ as $\mathbb{C} / \Lambda$. It follows that this gives a family of bidifferentials associated to any family of elliptic curves. In particular, this gives a bidifferential on the universal family over $Y(8)$.

Having chosen coordinates and a bidifferential, we get a (flat) connection on the pullback of $\mathbb{V}_{\lambda}$ to $Y(8)$. Since all our data is given explicitly, we may use classical properties of $\vartheta$ functions and modular curves to explicitly work out all terms involved in the discussion in Section 2.3 and Proposition 3.1 to compute the degree of the pullback of $\mathbb{V}_{\lambda}$ to $X(8)$ as in the $g=0$ case, hence the degee of $\mathbb{V}_{\lambda}$. This is not difficult, however, for the sake of variety we use a somewhat different argument which gives, as a byproduct, a simpler formula in the case of $\mathfrak{s l}_{2}$. 
From the existence of the flat connection on the pullback of $\mathbb{V}_{\lambda}$ to $Y(8)$, the discussion in Section 2.3 and Proposition 3.1, it follows that

$$
\operatorname{deg}\left(\mathbb{V}_{\lambda}\right)=\frac{1}{2\left(\ell+h^{\vee}\right)} \cdot\left\{r_{\lambda}(\alpha \ell \operatorname{dim}(\mathfrak{g})+\beta c(\lambda))-\sum_{\mu \in P_{\ell}} c(\mu) r_{\left(\lambda, \mu, \mu^{*}\right)}\right\}
$$

where $\alpha, \beta$ are constants independent of $\mathfrak{g}$. Here the term $\ell \operatorname{dim}(\mathfrak{g})$ comes from the bidifferential, the term involving $c(\lambda)$ comes from the change of coordinate and the last term comes from Proposition 2.10. Note that the coefficient of the last term is determined by the fact that there is a unique boundary component in $\overline{\mathrm{M}}_{1,1}$.

We now show that $\alpha=\beta=1 / 12$ by considering the case $\mathfrak{g}=\mathfrak{s l}_{2}$. Let the weight $\lambda \in P_{\ell}$ corespond to the integer $i$, so $0 \leq i \leq \ell$. The factorisation formula shows that the bundle of conformal blocks is 0 unless $i$ is even in which case the rank is equal to $\ell+1-i$. This is because the $\mu$ which give rise to a non-zero summand correspond to integers $j$ in the range from $i / 2$ to $\ell-i / 2$.

Since the individual local summands always have rank 1 and for a weight $\mu$ corresponding to an integer $j$ we have $c(\mu)=\frac{j^{2}+2 j}{2}$ it follows that the summand coming from Proposition 2.7

$$
\sum_{\mu \in P_{\ell}} c(\mu) r_{\left(\lambda, \mu, \mu^{*}\right)}=\sum_{j=i / 2}^{\ell-i / 2} \frac{j^{2}+2 j}{2}
$$

The other terms are

$$
r_{\lambda} \alpha \ell \operatorname{dim}(\mathfrak{g})=3 \alpha \ell(\ell+1-i)
$$

and

$$
r_{\lambda} \beta c(\lambda)=\frac{\beta(\ell+1-i)\left(i^{2}+2 i\right)}{2}
$$

We have

$$
\begin{aligned}
\sum_{j=i / 2}^{\ell-i / 2} \frac{j^{2}+2 j}{2}= & \sum_{j=1}^{\ell-i / 2} \frac{j^{2}+2 j}{2}-\sum_{j=1}^{i / 2-1} \frac{j^{2}+2 j}{2} \\
= & \frac{(\ell-i / 2)(\ell+1-i / 2)(2 \ell-i+1)}{12}-\frac{(i / 2-1)(i / 2)(i-1)}{12} \\
+\frac{(\ell-i / 2)(\ell+1-i / 2)}{2}-\frac{(i / 2-1)(i / 2)}{2} & =\frac{(\ell-i / 2)(\ell+1-i / 2)(2 \ell-i+7)}{12}-\frac{(i / 2-1)(i / 2)(i+5)}{12}
\end{aligned}
$$

If $i=0$, this is $\ell(\ell+1)(2 \ell+7) / 12$. Since $h^{\vee}=2$ it follows that in this case we get

$$
\operatorname{deg}\left(\mathbb{V}_{0}\right)=\frac{1}{2(\ell+2)}(3 \alpha \ell(\ell+1)-\ell(\ell+1)(2 \ell+7) / 12)=\frac{1}{24(\ell+2)}(\ell(\ell+1)(36 \alpha-2 \ell-7) .
$$

Since $12 \operatorname{deg}\left(\mathbb{V}_{0}\right)$ must be an integer for any $\ell$ it follows that we must have $\alpha=1 / 12$. 
For an arbitrary $\lambda$ we therefore have

$$
\begin{aligned}
& -12\left\{r_{\lambda}(\alpha \ell \operatorname{dim}(\mathfrak{g})+\beta c(\lambda))-\sum_{\mu \in P_{\ell}} c(\mu) r_{\left(\lambda, \mu, \mu^{*}\right)}\right\} \\
= & (\ell-i / 2)(\ell+1-i / 2)(2 \ell-i+7)-(i / 2-1)(i / 2)(i+5)-3 \ell(\ell+1-i)-6 \beta(\ell+1-i)\left(i^{2}+2 i\right) \\
= & (\ell-i / 2)(\ell+1-i / 2)(2 \ell-i+7)-(i / 2-1)(i / 2)(i+5)-(\ell+1-i)\left(6 \beta i^{2}+12 \beta i+3 \ell\right) \\
= & (\ell-j)(\ell+1-j)(2 \ell-2 j+7)-(j-1)(j)(2 j+5)+(\ell+1-2 j)\left(24 \beta j^{2}+24 \beta j+3 \ell\right)
\end{aligned}
$$

where we have put $j:=i / 2$ in the last line. Since $12 \operatorname{deg}\left(\mathbb{V}_{\lambda}\right)$ is an integer, the expression, thought of as polynomial in $\ell$, must be divisible by $\ell+2$. Working modulo $\ell+2$, the last line above is equal to

$$
\begin{array}{r}
(\ell-j)(\ell+1-j)(3-2 j)-(j-1) j(2 j+5)+(1+2 j)\left(24 \beta j^{2}+24 \beta j+3 \ell\right) \\
\simeq(\ell-j)(-1-j)(3-2 j)-(j-1) j(2 j+5)+(1+2 j)\left(24 \beta j^{2}+24 \beta j+6\right) \\
\simeq(2+j)(1+j)(3-2 j)-(j-1) j(2 j+5)+(1+2 j)\left(24 \beta j^{2}+24 \beta j+6\right) \\
=\left(j^{2}+3 j+2\right)(3-2 j)-j\left(2 j^{2}+3 j-5\right)+\left(48 \beta j^{3}+72 \beta j^{2}+(24 \beta+12) j+6\right) \\
=-2 j^{3}-3 j^{2}+5 j+6-2 j^{3}-3 j^{2}+5 j+\left(48 \beta j^{3}+72 \beta j^{2}+(24 \beta+12) j+6\right) \\
=(48 \beta-4) j^{3}+(72 \beta-6) j^{2}+(24 \beta-2) j
\end{array}
$$

This polynomial must be zero modulo $\ell+2$ so we must also have $\beta=1 / 12$.

Corollary 6.2. For $\mathfrak{g}=\mathfrak{s l}_{2}$ and $\lambda \in P_{\ell}$ even

$$
\operatorname{deg}\left(\mathbb{V}_{\lambda}\right)=-\left(\frac{\lambda^{2}-3 \lambda \ell+2 \ell^{2}-\lambda+2 \ell}{24}\right) .
$$

Proof. This follows easily from the theorem using the computations made in its proof.

One can see from the formula that for $\mathfrak{s l}_{2}, \operatorname{deg}\left(\mathbb{V}_{\lambda}\right)$ is always negative if $\ell>0$. Based on many other computations, it seems that this holds for all Lie algebras $\mathfrak{g}$ (whenever $r_{\lambda}>0$ ) except for the following example.

Corollary 6.3. For all $g, n$ with $g>0$ and $n \geq 1$ if $g=0, \mathfrak{g}=\mathfrak{e}_{8}, \ell=1$ and $\overline{0}=(0, \ldots, 0)$, we have $\mathbb{V}_{g, n, \overline{0}} \cong \mathrm{L}^{\otimes 4}$, where $\mathrm{L}$ denotes the Hodge line bundle on $\overline{\mathrm{M}}_{g, n}$.

Proof. It is well known, and since $P_{1}=\{0\}$ follows easily from the factorisation formula, that $r_{\overline{0}}=1$ for all $g, n$ as above. It thus suffices to compute the degree on all $\mathrm{F}$-curves. If we have an F-curve of type $\overline{\mathrm{M}}_{0,4}$ then again using $P_{1}=\{0\}$ the degree is easily seen to be zero.

Now consider the case $(g, n)=(1,1)$ and apply Theorem 6.1 . We have $\operatorname{dim}\left(\mathfrak{e}_{8}\right)=248$ and $h^{\vee}=30$ so it follows that in this case $\operatorname{deg}\left(\mathbb{V}_{0}\right)=1 / 3$. Since $\operatorname{deg}(\mathrm{L})=1 / 12$ and $\operatorname{Pic}\left(\overline{\mathrm{M}}_{1,1}\right)$ is a free abelian group generated by L, the claim follows in this case.

For general $g, n$ it follows from the propagation of vacuum that it suffices to consider the cases $(g, n)=(1,1)$ or $(g, n)=(g, 0), g>1$. It is also well known that $\operatorname{Pic}\left(\overline{\mathrm{M}}_{g, n}\right)$ is torsion free. Since $\mathbb{V}_{g, n, \overline{0}}$ is trivial on all F-curves of type $\overline{\mathrm{M}}_{0,4}$ it follows that $\mathbb{V}_{g, n, \overline{0}}$ is a power of L. To compute the precise power if $g>1$, one reduces to the $(1,1)$ case by restricting to a family of stable curves of arithemtic genus $g$ obtained by gluing a fixed smooth curve of genus $g-1$ to a varying family of 1-pointed curves of genus 1 and then using the factorisation formula. 
Remark 6.4. The construction of the bundles of conformal blocks provides a natural section of $\mathbb{V}_{g, n, \overline{0}}$. For $\mathfrak{g}=\mathfrak{e}_{8}$ and $g>0$, using some observations of Faltings [5, p. 12] this can be shown to be equal, upto a scalar, to the pullback via the Torelli map of the theta series associated to the $E_{8}$ lattice (which is a section of the fourth tensor power of the Hodge line bundle over $\mathrm{A}_{g}$, the moduli stack of principally polarised abelian vareties).

6.2. Nef bundles in higher genus. As we have noted, the bundles of conformal blocks are often not nef on $\overline{\mathrm{M}}_{g, n}$ if $g>0$. However, there is a canonical way of associating a nef divisor class on $\overline{\mathrm{M}}_{g, n}$ to any $\bar{\lambda} \in P_{\ell}^{n}$.

Proposition 6.5. For any $g \geq 0$ and $\bar{\lambda} \in P_{\ell}^{n}$, there is a unique $t \in \mathbb{Q}$ such that $c_{1}\left(\mathbb{V}_{g, n, \bar{\lambda}}\right)+$ tc $c_{1}(\mathrm{~L})$ is nef on $\overline{\mathrm{M}}_{g, n}$ and is trivial on any family of elliptic tails.

Proof. By the functoriality of conformal blocks, the factorisation formula and Lemma 2.5 it follows that $\mathbb{V}_{g, n, \bar{\lambda}}$ has non-negative degree on any $\mathrm{F}$-curve of $\mathrm{M}_{0,4}$ type. It also has constant degree on any family of elliptic tails since any such family gives the same class in $N_{1}\left(\overline{\mathrm{M}}_{g, n}\right)$. On the other hand $\mathrm{L}$ has degree zero on all $\mathrm{F}$-curves of $\mathrm{M}_{0,4}$ type and has a (constant) positive degree on any family of elliptic tails. It follows that there is a unique $t \in \mathbb{Q}$ (depending on $\bar{\lambda}$ and $g$ ) such that $c_{1}\left(\mathbb{V}_{g, n, \bar{\lambda}}\right)+t c_{1}(\mathrm{~L})$ is F-nef and trivial on any family of elliptic tails.

Using the functoriality of conformal blocks, the factorisation formula and Lemma 2.5 again, it follows that the restriction of $\mathbb{V}_{g, n, \bar{\lambda}}$ to the locus of flag curves is generated by sections, so has nef first Chern class. Since L restricts to a trivial bundle on the locus of flag curves it follows from $[10,(0.3)]$ that $c_{1}\left(\mathbb{V}_{g, n, \bar{\lambda}}\right)+t c_{1}(\mathrm{~L})$ is nef on $\overline{\mathrm{M}}_{g, n}$.

\section{Questions}

We conclude this paper by discussing some natural questions concerning the bundles of conformal blocks.

Question 7.1. Given a simple Lie algebra $\mathfrak{g}$ and an integer $n \geq 4$, is the closure of the subcone of $N^{1}\left(\overline{\mathrm{M}}_{0, n}\right)$ generated by determinants of conformal blocks for $\mathfrak{g}$ and all levels $\ell$ finitely generated? If so, is there an algorithm for computing this cone?

We do not know what to expect. For $n=5$ the determinants of conformal blocks for $\mathfrak{s l}_{2}$ generate the nef cone. This does not appear to hold for $n=6$, in which case computer calculations $^{2}$ suggest that the cone generated by conformal blocks for $\mathfrak{s l}_{2}$ has 128 vertices, 127 coming from the critical level and the remaining vertex corresponding to $\bar{\lambda}=(1,1,1,1,1,1)$ and level 1.

Question 7.2. Given an integer $n$, do the determinants of conformal blocks for all simple Lie algebras and all levels $\ell$ generate the nef cone of $\overline{\mathrm{M}}_{0, n}$ ?

We do not know if this is true for any $n \geq 6$. However, for $n=6$ the cone generated by conformal blocks for both $\mathfrak{s l}_{2}$ and $\mathfrak{s l}_{3}$ appears to strictly contain the cone for only $\mathfrak{s l}_{2}$.

One may ask a similar question for $\overline{\mathrm{M}}_{0, n} / S_{n}$.

For $g>0$, the procedure we described at the beginning of $\S 6$ shows that the Chern classes in cohomology lie in the subring generated by the classes of divisors. We therefore ask the following:

\footnotetext{
${ }^{2}$ These were carried out in part using polymake [8] .
} 
Question 7.3. Do the Chern classes in $\mathrm{CH}^{*}\left(\overline{\mathrm{M}}_{g, n}\right)_{\mathbb{Q}}$ of all conformal blocks bundles lie in the subring generated by divisors? If not, do they all lie in the tautological subring?

The second part of the question was suggested by Brendan Hassett. It seems likely that it has a positive answer.

\section{REFERENCES}

[1] T. ABE, Strange duality for parabolic symplectic bundles on a pointed projective line, Int. Math. Res. Not. IMRN, (2008), pp. Art. ID rnn121, 47.

[2] V. Alexeev and D. Swinarski, Nef divisors on $\bar{M}_{0, n}$ from GIT. arXiv.0812.0778.

[3] N. Bourbaki, Éléments de mathématique. Fasc. XXXIV. Groupes et algèbres de Lie. Chapitre IV: Groupes de Coxeter et systèmes de Tits. Chapitre V: Groupes engendrés par des réflexions. Chapitre VI: systèmes de racines, Actualités Scientifiques et Industrielles, No. 1337, Hermann, Paris, 1968.

[4] H. Esnault and E. Viehweg, Logarithmic de Rham complexes and vanishing theorems, Invent. Math., 86 (1986), pp. 161-194.

[5] G. Faltings, Theta divisors on moduli spaces of bundles. http://www.dmv2006.unibonn.de/vortraege/faltings.pdf.

[6] E. Frenkel And D. Ben-Zvi, Vertex algebras and algebraic curves, vol. 88 of Mathematical Surveys and Monographs, American Mathematical Society, Providence, RI, second ed., 2004.

[7] The GAP Group, GAP - Groups, Algorithms, and Programming, Version 4.4.12, 2008.

[8] E. GaWrilow AND M. Joswig, polymake: a framework for analyzing convex polytopes, in Polytopes - Combinatorics and Computation, G. Kalai and G. M. Ziegler, eds., Birkhäuser, 2000, pp. 43-74.

[9] N. Giansiracusa, Conformal blocks and rational normal curves. arXiv:1012.4835.

[10] A. Gibney, S. Keel, And I. Morrison, Towards the ample cone of $\bar{M}_{g, n}$, J. Amer. Math. Soc., 15 (2002), pp. 273-294 (electronic).

[11] B. Hassett, Moduli spaces of weighted pointed stable curves, Adv. Math., 173 (2003), pp. 316-352.

[12] M. M. Kapranov, Chow quotients of Grassmannians. I, in I. M. Gel'fand Seminar, vol. 16 of Adv. Soviet Math., Amer. Math. Soc., Providence, RI, 1993, pp. 29-110.

[13] S. KEEL, Intersection theory of moduli space of stable n-pointed curves of genus zero, Trans. Amer. Math. Soc., 330 (1992), pp. 545-574.

[14] S. Keel and J. McKernan, Contractible extremal rays on $\bar{M}_{0, n}$. alg-geom/9607009.

[15] P. Littelmann, Characters of representations and paths in $\mathfrak{H}_{\mathbf{R}}^{*}$, in Representation theory and automorphic forms (Edinburgh, 1996), vol. 61 of Proc. Sympos. Pure Math., Amer. Math. Soc., Providence, RI, 1997, pp. 29-49.

[16] E. LoOIJEnGA, Conformal blocks revisited. math.AG/0507086.

[17] D. Mumford, Tata lectures on theta. I, vol. 28 of Progress in Mathematics, Birkhäuser Boston Inc., Boston, MA, 1983. With the assistance of C. Musili, M. Nori, E. Previato and M. Stillman.

[18] J. H. Silverman, The arithmetic of elliptic curves, vol. 106 of Graduate Texts in Mathematics, Springer-Verlag, New York, 1986.

[19] C. Sorger, La formule de Verlinde, Astérisque, (1996), pp. Exp. No. 794, 3, 87-114. Séminaire Bourbaki, Vol. 1994/95.

[20] Y. Tsuchimoto, On the coordinate-free description of the conformal blocks, J. Math. Kyoto Univ., 33 (1993), pp. 29-49.

[21] A. Tsuchiya And Y. Kanie, Vertex operators in conformal field theory on $\mathbf{P}^{1}$ and monodromy representations of braid group, in Conformal field theory and solvable lattice models (Kyoto, 1986), vol. 16 of Adv. Stud. Pure Math., Academic Press, Boston, MA, 1988, pp. 297-372.

[22] A. Tsuchiya, K. Ueno, And Y. Yamada, Conformal field theory on universal family of stable curves with gauge symmetries, in Integrable systems in quantum field theory and statistical mechanics, vol. 19 of Adv. Stud. Pure Math., Academic Press, 1989, pp. 459-566.

[23] K. Ueno, Introduction to conformal field theory with gauge symmetries, in Geometry and physics (Aarhus, 1995), vol. 184 of Lecture Notes in Pure and Appl. Math., Dekker, 1997, pp. 603-745. 
School of Mathematics, Tata Institue of Fundamental Research, Homi Bhabha Road, Mumbai 400005, India

E-mail address: naf@math.tifr.res.in 